\title{
Numerical Modeling of Core Compressor Discharge for a Windmilling Aero Engine
}

\author{
Luis E. Ferrer-Vidal ${ }^{1}$ \\ Cranfield University \\ College Road, Cranfield, Bedfordshire, MK43 0AL, U.K. \\ l.ferrer-vidal@cranfield.ac.uk

\section{Vassilios Pachidis} \\ Cranfield University \\ College Road, Cranfield, Bedfordshire, MK43 0AL, U.K. \\ v.pachidis@cranfield.ac.uk
}

\begin{abstract}
A gas turbine engine's ability to relight in flight depends heavily on the combustion chamber's inlet conditions. These conditions are dictated by the core compression system located immediately upstream of the combustor, but such conditions during windmill have not been thoroughly investigated and are not commonly available in the literature. In this paper, an entire high pressure ratio axial compressor is modelled using scale adaptive simulation. Real windmilling inlet conditions extracted from altitude test facility data are used as boundary conditions. The implications of steady vs. unsteady modelling and the use of different interface modelling approaches are investigated. A description of the discharge flow-field is given in terms of flow statistics, turbulent quantities, and dominant dynamic modes. The discharge flow-field is dominated by small scale structures resulting from leading edge separation in the last stator (OGV), with little influence of the upstream stages on the stator flow-field dynamics. The study has shown that, while an unsteady analysis is required, reduced
\end{abstract}

\footnotetext{
${ }^{1}$ Corresponding author.
} 
order models allowing for a decoupled analysis of the last stage flow dynamics may be sufficient in future studies seeking to characterize combustor inlet conditions for inflight relight.

\section{KEYWORDS: Sub-idle, Windmilling, Compressor, Scale Adaptive Simulation INTRODUCTION}

Engine flame-out is rare but may occur due to a variety of reasons including compressor surge, weather conditions (rain or hail ingestion), volcanic ash, bird strikes, crew action, or an incorrect idle trim. Perhaps the most common failed-relight scenario is due to flight in rain and hail conditions, where both engines flame out and either fail to relight or are not able to accelerate past idle in an acceptable amount of time [1]-[3]. To mitigate the risk of these events, regulating agencies have established inflight relight performance requirements which new engines must meet for certification [4]. The ability to meet these requirements may become more challenging in the future as engine bypass ratios increase to improve propulsive efficiency, resulting in smaller core mass flows that put a strain on the combustor's ability to relight. In order to improve our understanding of relight performance and ensure new designs meet requirements, an accurate understanding of combustor relight performance is required, and this in turn requires an appropriate understanding of the core compressor discharge conditions that feed the combustor during an inflight relight event.

Inflight relights may be attempted with the aid of the starter or from a free windmilling condition depending on the altitude and airspeed, which dictate compressor RPM and combustor conditions. This work considers a relight at the "top- 
left corner" of the windmill envelope, i.e. the point at highest altitude and lowest airspeed at which a relight may be attempted without aid of the starter. This constitutes the most challenging conditions for a relight due to the combination of low air density and mass flow. The location of this operating point on a generic relight envelope is schematically shown in Figure 1.

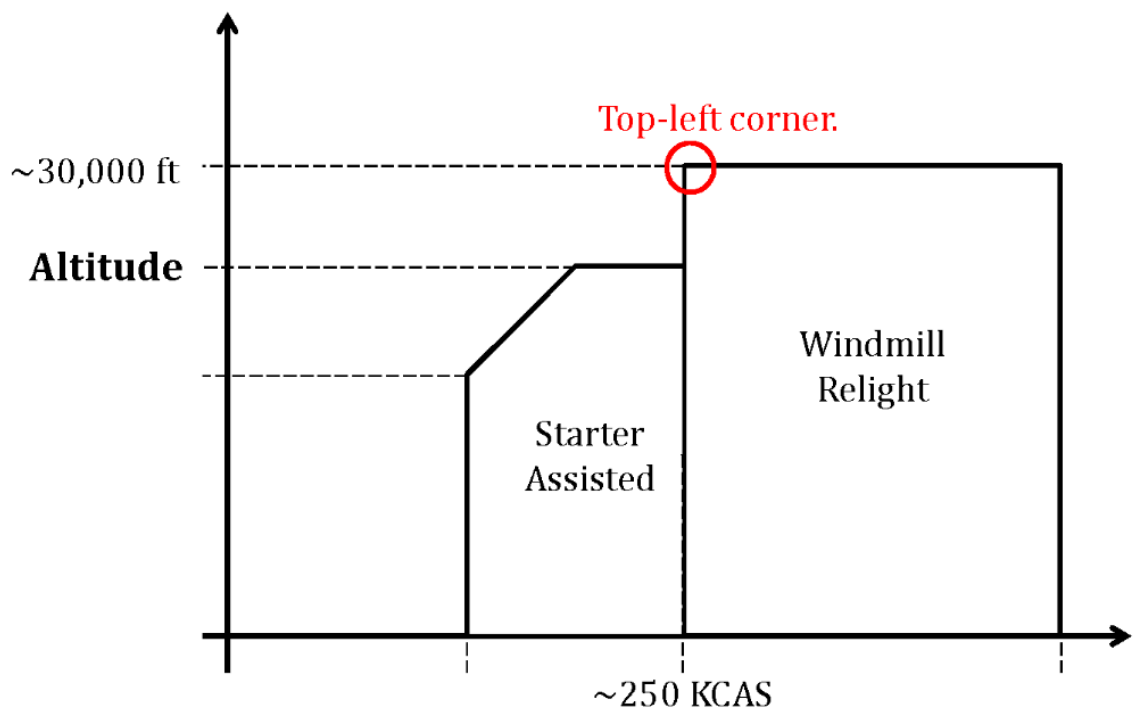

Airspeed

Figure 1 Generic relight envelope showing starter assisted and windmill relight regions and location of "top left corner" condition investigated in this work.

There has been research on combustor relight at altitude [5][6] and work on characterizing the discharge conditions of new generation combustors [7]. These investigations have had to rely on bulk temperature and pressure values obtained from empirical knowledge of the operating conditions to establish their combustor inlet boundary conditions. The authors have not been able to find a study where detailed core compressor discharge conditions have been used to model combustor 
relight performance at altitude. Since flow-field details and dynamics at the combustor pre-diffuser inlet may be relevant to fuel atomization performance [8], core compressor discharge conditions may be an important factor in determining an engine's ability to relight. An illustrative example is the one existing study of compressor discharge conditions into the combustor during windmill [8]. In that work, negative incidence flow on the compressor OGV was shown to result in the formation of a strong jet that could be exploited to improve fuel atomization at low windmilling core mass flows. That study however relied on steady state numerical analysis, with possible unsteady effects not considered. Therefore, the first objective of this work is to characterize compressor discharge conditions at windmill so they may be used for combustion relight studies. The second objective is to assess compressor modelling methods for these conditions. Since core compressors consist of multiple stages, special attention must be given to the way blade-row interfaces are modelled, since these may impact the calculated flow dynamics. An understanding of the modelling requirements can then be used for further studies requiring the generation of further compressor exit profiles for combustion modelling at similar off-design conditions.

\section{Interface Modelling}

One of the challenges with turbomachinery CFD is how to handle the interface between the rotating and non-rotating components [10]. Different approaches are warranted depending on the application and whether an unsteady solution is sought.

There are two methods commonly used for steady state approaches: 
- Mixing Planes: Where the flow is circumferentially averaged in bands at each interface and passed onto the next blade row [11].

- Frozen Rotor: Where a frame of reference change is made to account for the rotor velocity, but the circumferential position of components is fixed.

Both methods have their application. Mixing planes can be considered a good approximation to the time-averaged unsteady case, but cannot be used for problems exhibiting large circumferential variation. The frozen rotor approach can be considered a good steady-state approximation for steady modeling of flows with large circumferential variations, but should not be used in cases where the quasi-steady assumption regarding rotor movement does not apply [11]. With the frozen rotor approach, the different pitch ratio between turbomachinery components (caused by differing blade counts) is handled by flux scaling. Errors due to scaling can be mitigated by modeling more than a single passage until the pitch ratio is brought close to unity, though this may require large computational expense for multiple stage machines [4].

The mixing plane and frozen rotor approaches can still be applied to unsteady cases. Such approaches would consider unsteadiness within the passage, but would not capture rotor-stator interaction. In order to do so, a variety of models exists which we shall term transient rotor-stator (TRS) interface models. The difference between different TRS models is due to how each handles the issue of unequal blade counts in order to avoid having to model an entire annulus [12]-[14]. The most prevalent of these methods is the Profile Transformation method. This method handles the 
unequal pitch by scaling in the same manner as the frozen rotor approach, but considering the moving wake due to the unsteady formulation [11]. Time transformation [12] and Fourier transformation [13] methods also exist as alternatives to the profile transformation method. The time transformation method typically needs to be used in combination with profile transformation to model multistage machines beyond 1.5 stages, while the Fourier transformation method is not currently available for models with multiple stages [11]. These reasons coupled to the perfect pitch matching in the last stage of the analyzed geometry led this study to consider only profile transformation.

\section{Unsteady Modeling}

Unsteady Reynolds Average Navier Stokes (URANS) modelling is the unsteady extension of the RANS approach, where the time-derivative term is included in the RANS equations. This approach is appropriate for most unsteady cases where the flow unsteadiness is driving the flow, such as time-dependent boundary conditions. For windmilling compressors, there may be considerable flow unsteadiness arising from flow separation and recirculation due to high negative incidence flows [15]. This raises the question of the "spectral gap" or "separation of scales" problem when attempting to use URANS to characterize such flows [16]-[19]. This is due to RANS being conceptually formulated to account for the ensemble average effect of all small scale turbulent fluctuations, with URANS considered as a way to capture unsteady dynamics of much larger time-scale than the underlying turbulence. 
Scale adaptive simulation (SAS) forms part of a class of so-called scale resolving methods that allow turbulent spectrum resolution in those parts of the flow that become unstable, while still keeping a URANS formulation in stable regions [20]-[22]. SAS models can be considered improved URANS models that modify the turbulence length scale equation, (i.e. the omega equation in SST) through inclusion of the von Karman length scale [20], defined as:

$$
L_{v K}=\frac{\kappa S}{\left|U^{\prime \prime}\right|}
$$

Where $\kappa$ is a constant, $S$ the strain rate and $U^{\prime \prime}$ the second derivative of velocity.

In its implementation, a limiter on $L_{v K}$ is used which is proportional to cell size, making the resolved turbulence also a function of the mesh refinement, making the behaviour somewhat similar to a LES model [21]. The $L_{v K}$ term is used within the omega equation of the SST formulation to increase the dissipation of modelled TKE in those regions where the flow instability can be resolved, avoiding over-damping that could otherwise arise from the RANS formulation. The SAS method thus allows RANS meshes to be used in unsteady analyses that may give rise to flow instabilities. SAS has been gaining popularity for turbomachinery and other complex industrial problems requiring scale resolving capability [23]-[25].

\section{METHODS}

The three-dimensional compressible RANS equations are solved in ANSYS CFX, a commercial pressure-based coupled solver. The compressible RANS equations are density weighted, constituting a Favre average, though this distinction is of little 
importance at the low speeds investigated. The governing equations are linearized via a finite volume discretization using a co-located (non-staggered) grid (same grid for all transport equations) [26]. In CFX, the discretization is implicit and the problem behaviour is kept hyperbolic via the inclusion of the time derivative term, even for steady-state analysis. This entails that the steady-state time step acts as an acceleration parameter to drive the solution to a converged steady state (pseudo time step). For unsteady cases, an additional inner iteration loop on the linear solver is used to achieve convergence at a given time-step before moving onto the next, ensuring temporal accuracy. Steady state analysis is used to initialize URANS and SAS solutions. SST is the underlying RANS model chosen for this work due to its suitability to internal flows [11].

\section{Boundary conditions}

Altitude test facility data for a "top-left corner" windmill relight for a modern turbofan engine has been provided by the sponsor. The first ten seconds of the transient data correspond to a stabilized windmill condition. Engine station data are time-averaged for those ten seconds to obtain a set of boundary conditions in terms of inlet total temperature and pressure, outlet static pressure, and shaft RPM for the high pressure compressor. While the instrumentation was duly calibrated, uncertainty ranges for the transient data are unknown so a study of their impact on the results could not be carried out, however given the separated flow field inside the machine, it is unlikely these would greatly impact results at the discharge plane. The comparative study of the applied methods would not be affected. 
The compressor in question consists of six stages and is modelled with a mid-stage handling bleed to account for the open bleed valves that would be expected during sub-idle operation. A mass flow outlet boundary condition is used for the bleed in order to fix a constant outlet flow fraction in terms of the compressor inlet flow. Inlet total pressure and temperature and outlet static pressure are prescribed to match the test data.

\section{Temporal Discretization}

The temporal discretization scheme used is Second Order Backward Euler, which is an implicit second-order accurate scheme [11]. Table 1 shows the timescales associated with the OGV for the windmilling case under consideration. The final selected time step is also shown. The selected time step corresponds to a circumferential translation of $\sim 2$ mesh elements per time step. Adaptive time stepping was initially used to arrive to this time step. CFX adaptive time stepping allows a convergence criteria to be set and the time step varied to ensure this convergence criteria is reached at each time step, enabling a time step and convergence criteria analysis to be performed concurrently [26]. The convergence criteria used was $1 \mathrm{E}-5$ normalized residuals on all conservation equations and stable bulk performance parameters (overall pressure ratio and torque). A separate run was performed with a time step of $1 \mathrm{E}-7$ s, yielding normalized residuals of 1E-6. Figure 2 shows a comparison of velocity traces at a rake downstream of the last stator for both time steps.

Table 1: OGV time-scales during stabilized windmill. 


\begin{tabular}{|l|l|}
\hline Mid-span Chord Convective Time (OGV blade only) & $5.1 \times 10^{-4} S$ \\
\hline Pitch Passing Period & $4.6 \times 10^{-4} S$ \\
\hline RANS Turbomachinery "Best Practice" $(0.1 / \Omega)[11]$ & $5.2 \times 10^{-4} S$ \\
\hline Locked Blade Shedding Period (St=0.6) $[28]$ & $8.5 \times 10^{-4} S$ \\
\hline Selected Time Step & $1.0 \times 10^{-6} S$ \\
\hline
\end{tabular}

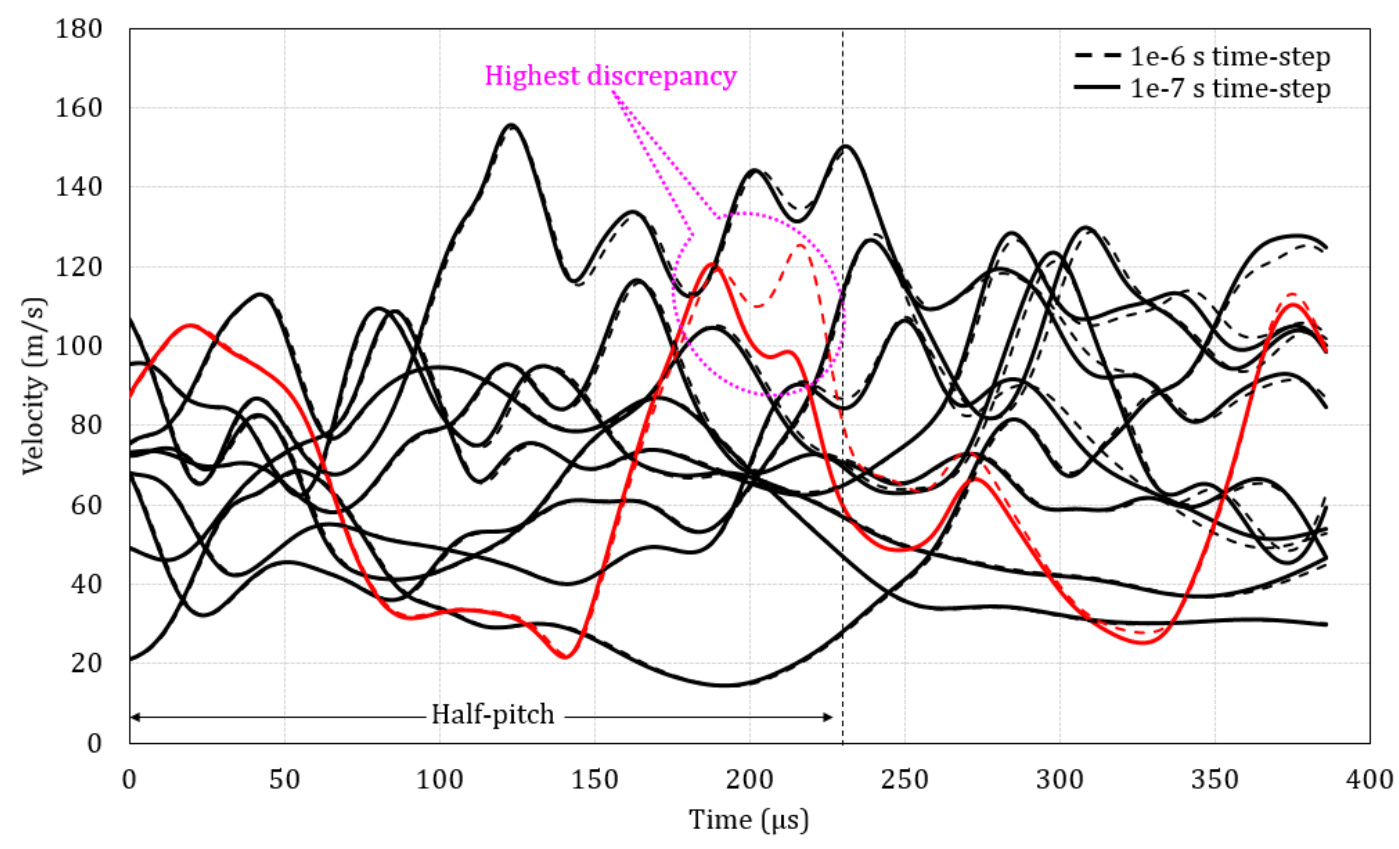

Figure 2 Timestep sensitivity of calculated velocity traces on a rake downstream of last stator.

The convective time from R1 to S6 (inlet to outlet of compressor) is $9.79 \mathrm{E}-3$ s so that a full pass-through of the entire compressor geometry requires 10000 time steps using the selected time step. The selected time-step yields a CFL number below 1 in the region of interest as shown in Figure 3, ensuring a time-accurate calculation. The maximum CFL number in the entire model is 4.7.The red region near the leading edge is associated to small length-scales near the leading edge. 


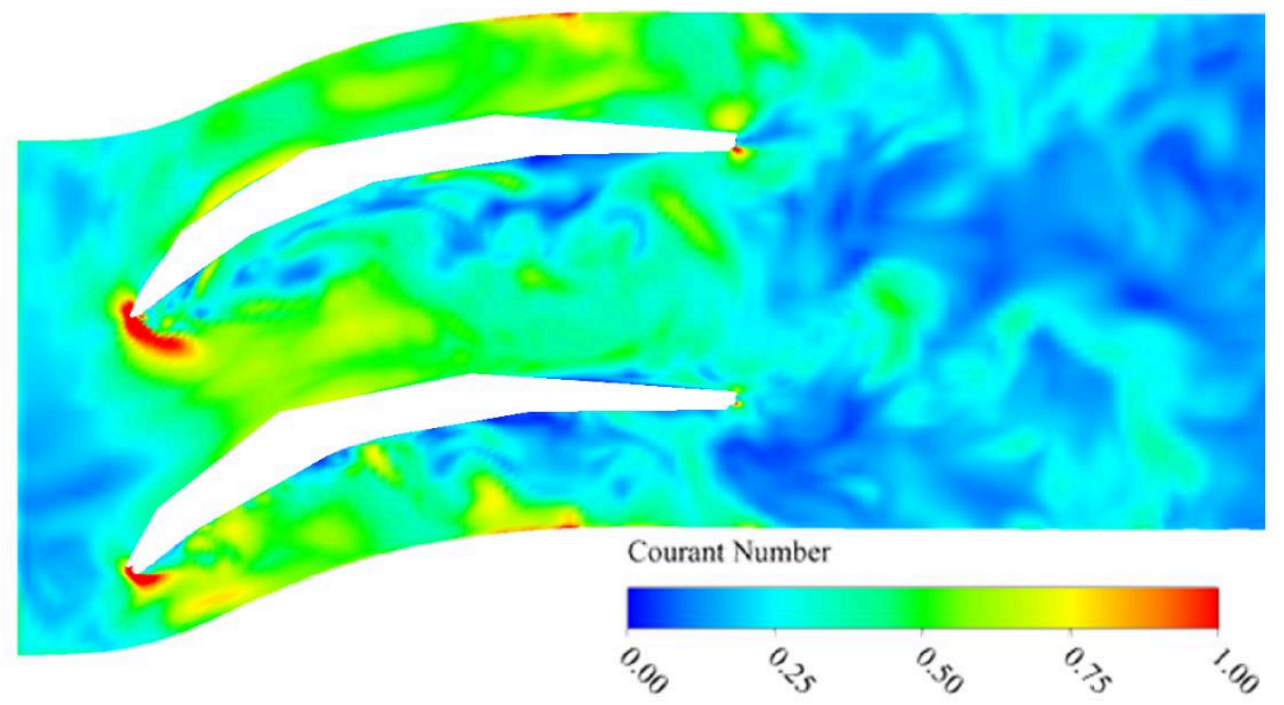

Figure 3 CFL on stator domain of interest. Geometry altered due to confidentiality. [Color online].

\section{Spatial Discretization}

The geometry has been discretized using a structured mesh using ANSYS Turbogrid. A mesh independent RANS solution is used for initialization, following the study in [15]. Each blade-row domain upstream of the last stage in base-line RANS mesh consists of approximately 70 spanwise elements, 70 circumferential elements and 100 axial elements for an approximate node count of $\sim 5 E 5$ per blade row. A y+ 30 is used and wall functions applied to model the near-wall region for the base-line RANS mesh. In order to ensure more adequate resolution, wall-resolved $(y+<2)$ meshes in the last stage have been used, resulting in node counts $\sim 1 \mathrm{E} 6$ for the last two blade-rows. While the concept of mesh independence cannot be applied to scale resolving models, as the level of resolved turbulence will be affected by the mesh size, adequate mesh sizing can be gauged by ensuring the resolved turbulence spectrum in the inertial range follows the $-5 / 3$ slope rule [27]. This has been checked in results. For SAS models, RANS sized meshes may be used [28][20]. Starting from the 
independent RANS mesh, the SAS meshes for the last stage have been refined by a factor of 2 in order to ensure sufficient turbulence resolution. Comparison of the different levels of turbulence resolution is given in Figure 4 in terms of the ratio of unresolved (modeled) turbulence kinetic energy $\left(k_{u}\right)$ to total turbulence kinetic energy ( $\left.k_{\text {total }}\right)$, where values of 1 indicate all the turbulence kinetic energy has been modeled and a value of 0.5 indicates an even split. Similar information may be obtained from the eddy viscosity ratio, which gives the ratio of modeled to molecular viscosity.

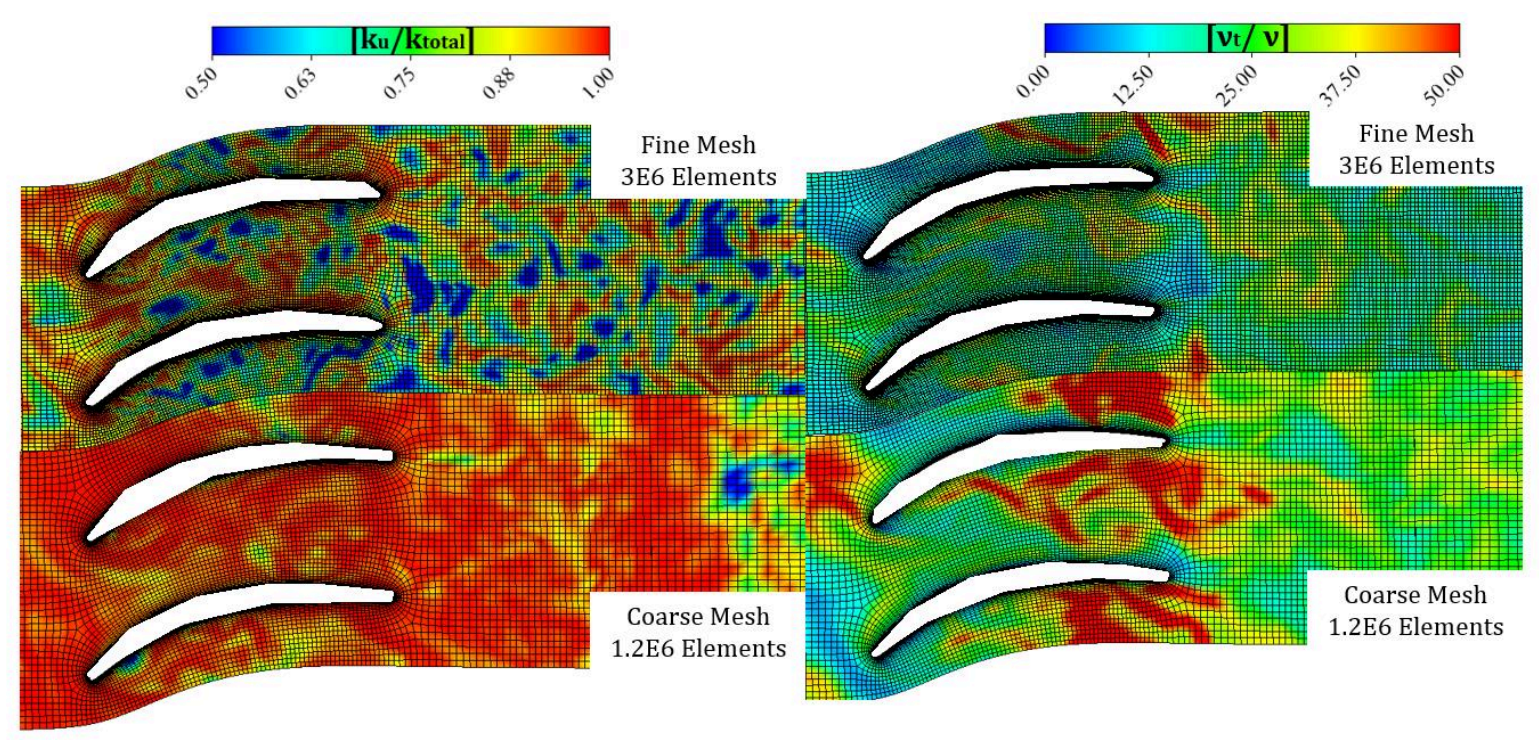

Figure 4 Turbulence resolution on RANS and refined SAS meshes. Left: Modeled to total TKE ratio. Right: Eddy viscosity ratio. Geometry altered due to confidentiality. [Color online].

The SAS model will adapt to the scales that can be resolved by the given mesh sizing and time-step. This will also depend on the length scales present in the flow-field. For a largely stable flow-field with only small scales present, the SAS will converge to a standard URANS implementation [20]. In the case of a windmilling compressor, we can expect the flow to remain mostly attached in front stages before separating in the 
rear stages [15]. The attached flow-field in upstream stages would be covered by the URANS formulation, with the turbulent-resolving term being triggered in the separated downstream stages. Figure 5 shows this for the windmilling case studied. Values of 1 indicate regions where SAS has not been triggered and all turbulence is modeled by the URANS SST model. Both instantaneous and time-averaged flow-fields are shown. As can be seen, the SAS model is triggering the turbulence resolution in the region of interest, namely the final stage. Note that while only one passage has been modelled for the last rotor shown in Figure 5, multiple instances are shown for ease of visualization of the only rotating components with TRS interfaces.

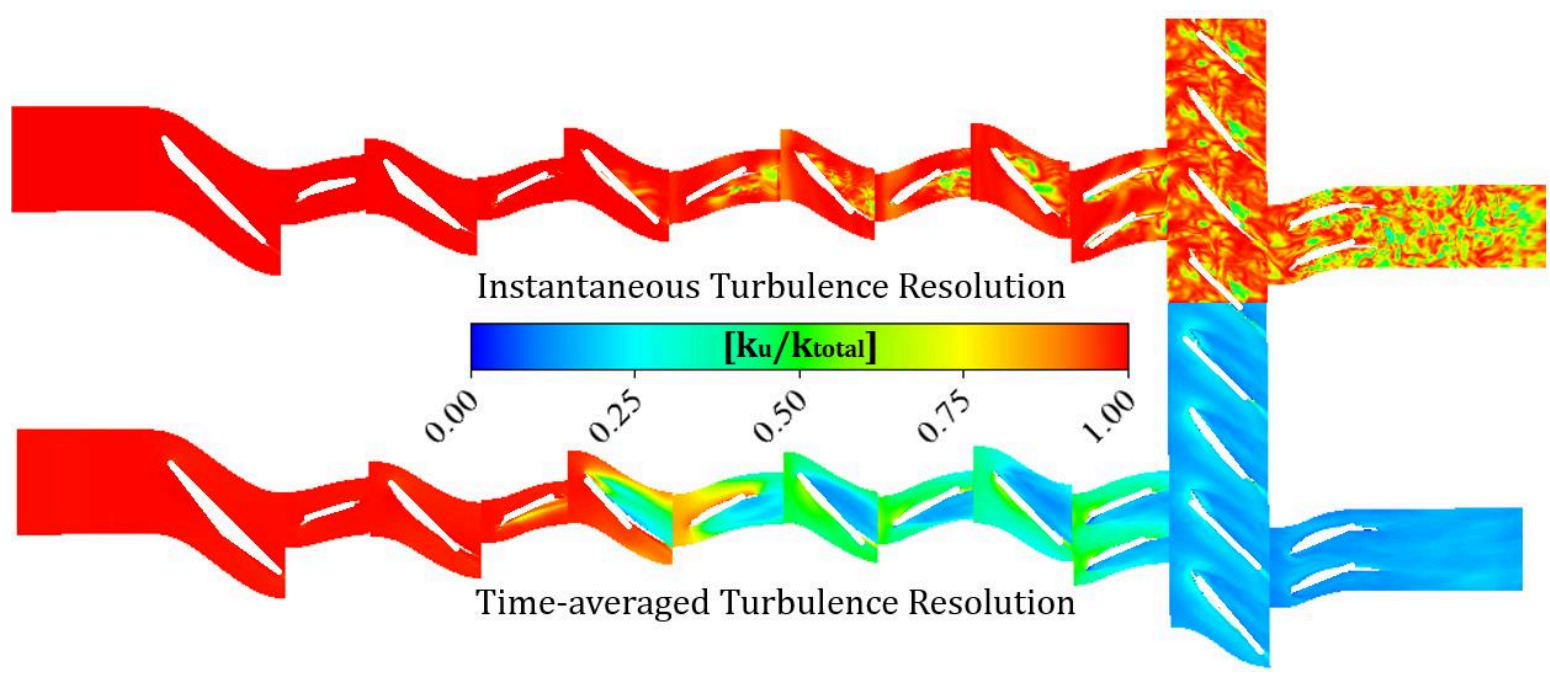

Figure 5 Time-averaged and instantaneous turbulence resolution on entire compressor geometry. Case shown corresponds to TRS interfaces applied only on last stator and mixing planes applied elsewhere. Geometry altered due to confidentiality. [Color online].

All analysis for this study has focused on the extraction of adequate boundary conditions at a discharge plane, located $\sim 7 \mathrm{~mm}$ downstream of the OGV. This plane represents the pre-diffuser inlet. Figure 6 shows the SAS mesh resolution as this plane as well as the coordinate convention to be used in the results. 


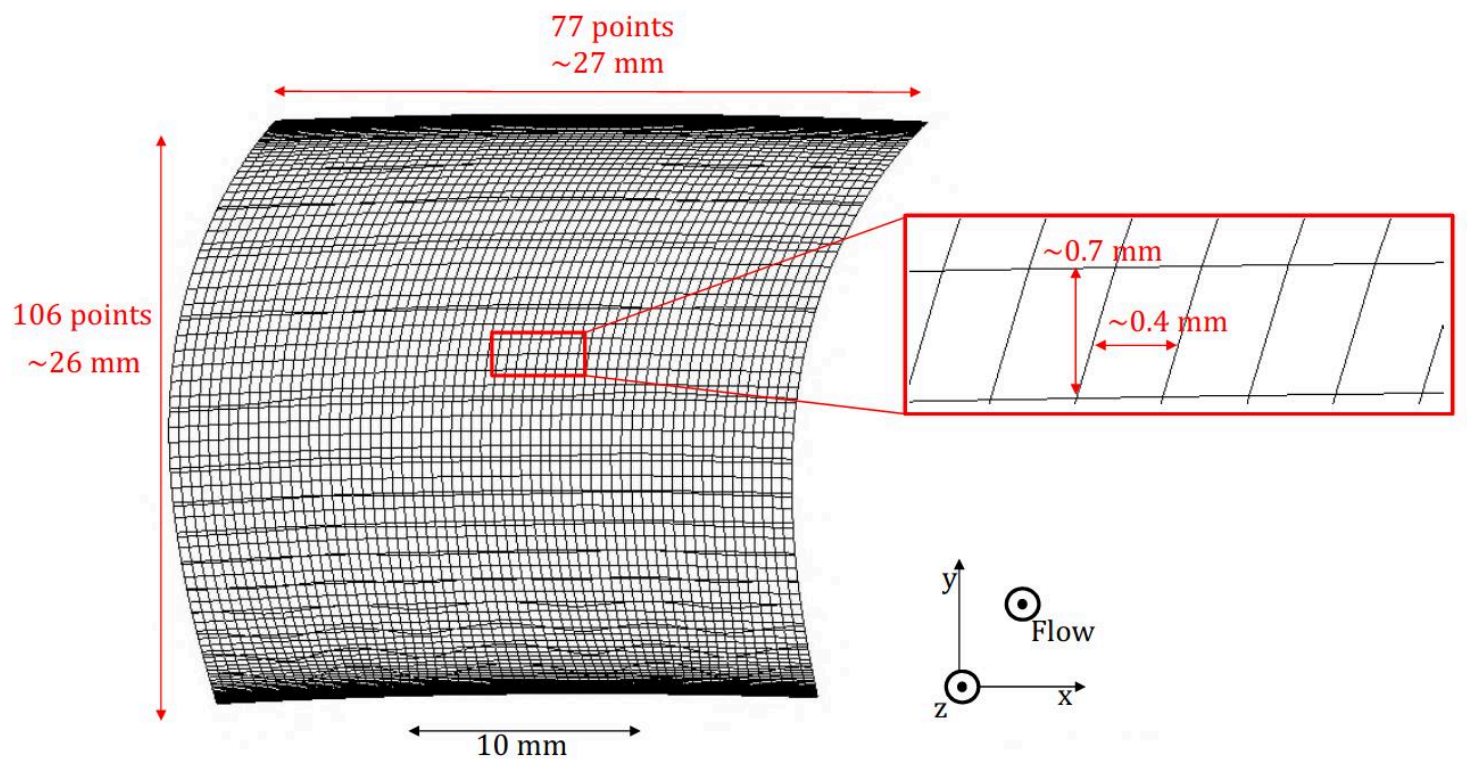

Figure 6 Mesh resolution at discharge plane of interest.

\section{Interface Modelling}

Due to the inherent unsteadiness in turbomachinery, the impact of domain interface models on results needs to be studied to determine the minimum model fidelity required. In this study, the effects of different interface plane setups have been studied by comparing the results from the different approaches as shown in Table 2 . An illustration comparing the "Full TRS" model and the"1.5 Stage TRS" model is shown in Figure 7.

Table 2 Different interface modelling approaches investigated.

\begin{tabular}{|l|l|}
\hline Model & Description \\
\hline Full TRS & $\begin{array}{l}\text { Transient rotor-stator (TRS) profile transformation interfaces } \\
\text { throughout. }\end{array}$ \\
\hline 1.5 Stage TRS & TRS only in last 1.5 stage, Mixing planes elsewhere. \\
\hline Mixing Planes & Mixing planes throughout. \\
\hline
\end{tabular}




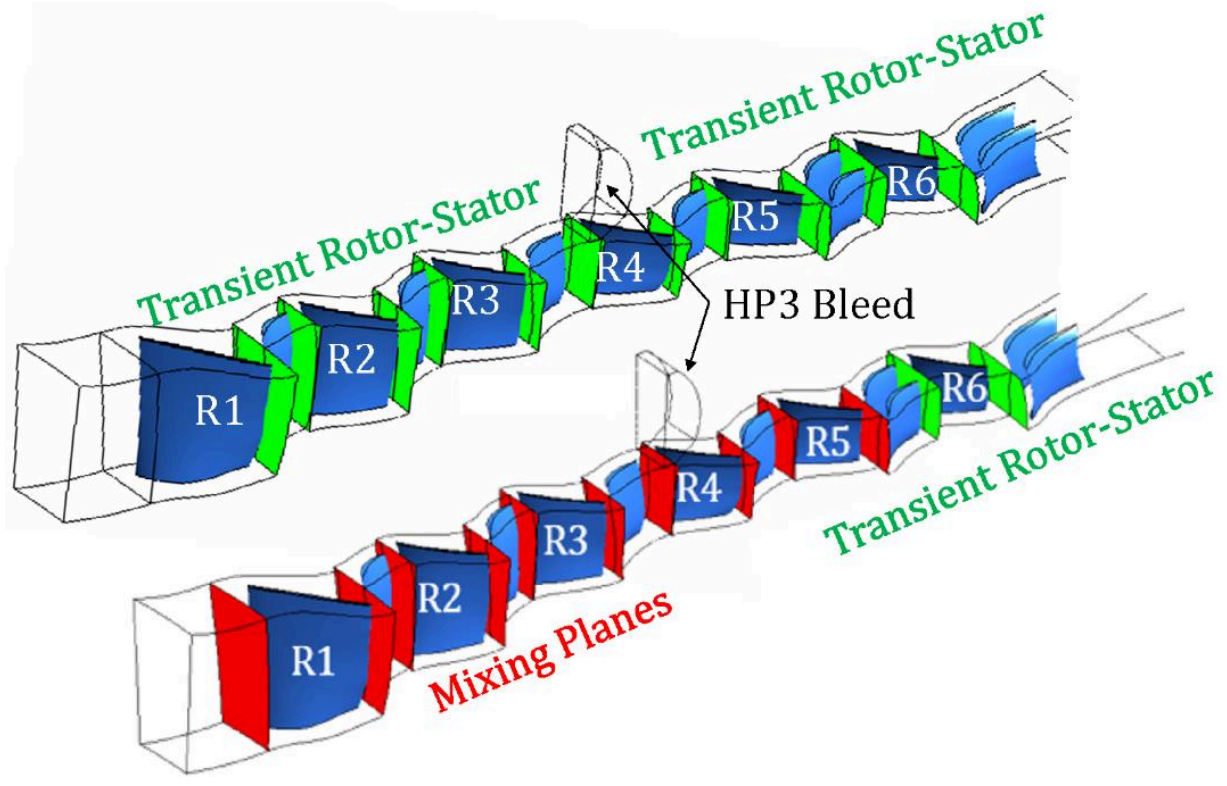

\section{Figure 7 Compressor model used. Two different approaches of applying TRS and mixing plane interfaces shown.}

\section{Post Processing}

Fourier analysis is used to extract turbulence spectra. The time series data used to do this has been extracted from five different radial points at the same axial location as the discharge plane of interest. Welch's method is implemented in MATLAB to obtain the power spectral density estimate of the signal. Via Welch's method, the signal is split into 8 segments with 50\% overlap and the resulting periodograms are averaged to obtain the estimate. A Hamming window is used to filter the periodograms and mitigate spectral leakage. This method is also used to help remove the initial transient from unsteady data by comparing the periodograms from each segment of the data: Initial periodograms with different power spectral densities indicate that the associated time frame is part of the initial transient and should be discarded.

Modal analysis is performed on the discharge plane of interest. This is done via proper orthogonal decomposition of the flow-field velocity fluctuations. The mean velocity is 
subtracted from data planes sampled every 10 computational time steps. The velocity fluctuation field at each plane is reshaped into a column vector, and a matrix built where each column corresponds to a sampled time step or "snapshot". Singular Value Decomposition of this matrix is performed in MATLAB, yielding the spatial modes ordered by magnitude of the corresponding singular values $\left(\sigma_{i}\right)$. The squared magnitude of the singular values corresponding to each mode constitute the associated signal power, and are a measure of the TKE associated to each mode. This can be used to calculate $E_{f r a c}$, the fraction of the total resolved TKE contained in each mode, or [30]:

$$
E_{\text {frac }_{i}}=\frac{\sigma_{i}^{2}}{\sum_{i} \sigma_{i}^{2}}
$$

\section{Sensitivity to Heat Soakage \& Tip Clearance}

The case studied has considered that thermal equilibrium exists between the compressor metal structure and the gas path. Adiabatic wall boundary conditions have thus been applied in the compressor model. This is a valid assumption for the stabilized windmill relight conditions which are of interest here. In a real relight at altitude, an aircraft at cruise will typically require a descent to lower altitudes before a relight can be attempted (due to air density constraints for combustion). During this descent, a stabilized RPM is established and thermal equilibrium is reached: the compressor is considered to be in a "cold-soaked" condition prior to a relight attempt. The sensitivity of the discharge conditions to this assumption is addressed here considering a worst case scenario where the compressor is assumed to maintain a 
wall temperature distribution matching the cruise gas path temperatures. This temperature is obtained from knowledge of the nominal axial temperature ratio distribution across the compressor during cruise and the compressor cruise inlet conditions at altitude, obtained from an in-house NPSS [29] whole-engine performance model. The compressor is assumed to reach thermal equilibrium at cruise and no metal cooling is assumed during deceleration. This is not realistic, but allows a worst case of the effects of heat soakage on the modeled conditions to be studied. While the effects of heat transfer could be disregarded for stabilized windmill, they would be much more relevant for a quick windmill relight, which is performed at lower altitudes immediately after flameout and before a steady operating point is reached. Quick windmill relight conditions are outside the scope of this work.

Blade tip clearances are often a source of uncertainty in compressor models, as the state of abradable casing liners is often unknown and hard to measure. The mechanical shaft speed and thermal state of the casing and blading also affect this parameter. In this work, a constant blade tip clearance of $1 \%$ span has been assumed for each rotor blade. A sensitivity study is also carried out where the tip clearance is eliminated and increased to $2 \%$ span respectively in order to assess its effect on the compressor discharge flow-field.

\section{RESULTS \& DISCUSSION}

This investigation seeks to characterize the compressor discharge conditions during windmill. How much this is affected by modeling choices is the first item to assess. This is followed by a description of the flow-field at the discharge plane of interest. 
Finally, a simple sensitivity analysis is performed to assess the impact that compressor heat soakage and tip clearance may have on the results presented in this work, and to compressor windmill discharge conditions in general.

\section{Unsteady Methods}

Comparison of steady state RANS, URANS SST and SAS SST shows the need to consider an unsteady analysis. While steady state bulk performance parameters (pressure ratio and torque) obtained from RANS converge to the performance predicted by time-averaged unsteady methods, Figure 8 shows that the flow-field predicted by the converged steady solution does not match that obtained from timeaveraged unsteady analyses. The figure also shows that URANS and SAS yield broadly equivalent time-averaged flow-fields.

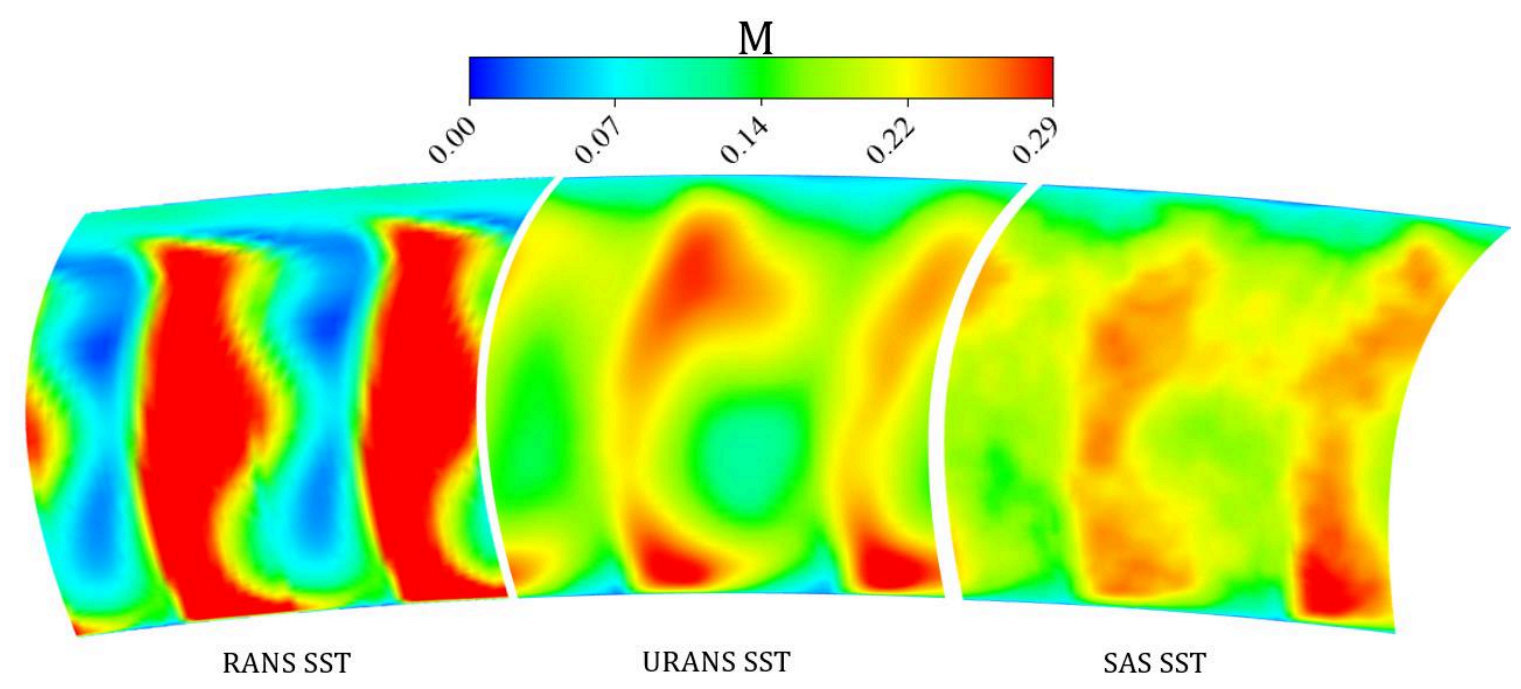

Figure 8 Time averaged velocity magnitude at Discharge Plane with RANS, URANS and SAS (all using the SST turbulence model). TRS interfaces used for URANS and SAS. [Color online].

A previous study [8] looked at compressor discharge conditions during windmill and determined that the formation of a strong axial jet due to flow separation could have 
the potential to improve fuel atomization in the injector. This could be expected to at least partially mitigate the detrimental effects of low temperature and flow during relight from a stabilized windmill. While that is also initially seen in the steady results in this study, which uses a different geometry than that in [8], the strength of this jet is seen to diminish when the unsteady solution is considered. Figure 9 compares the resulting discharge flow-fields in the compressor pre-diffuser. A strong separation region that is clearly visible at the top of the pre-diffuser in the RANS solution is not present in the unsteady result.

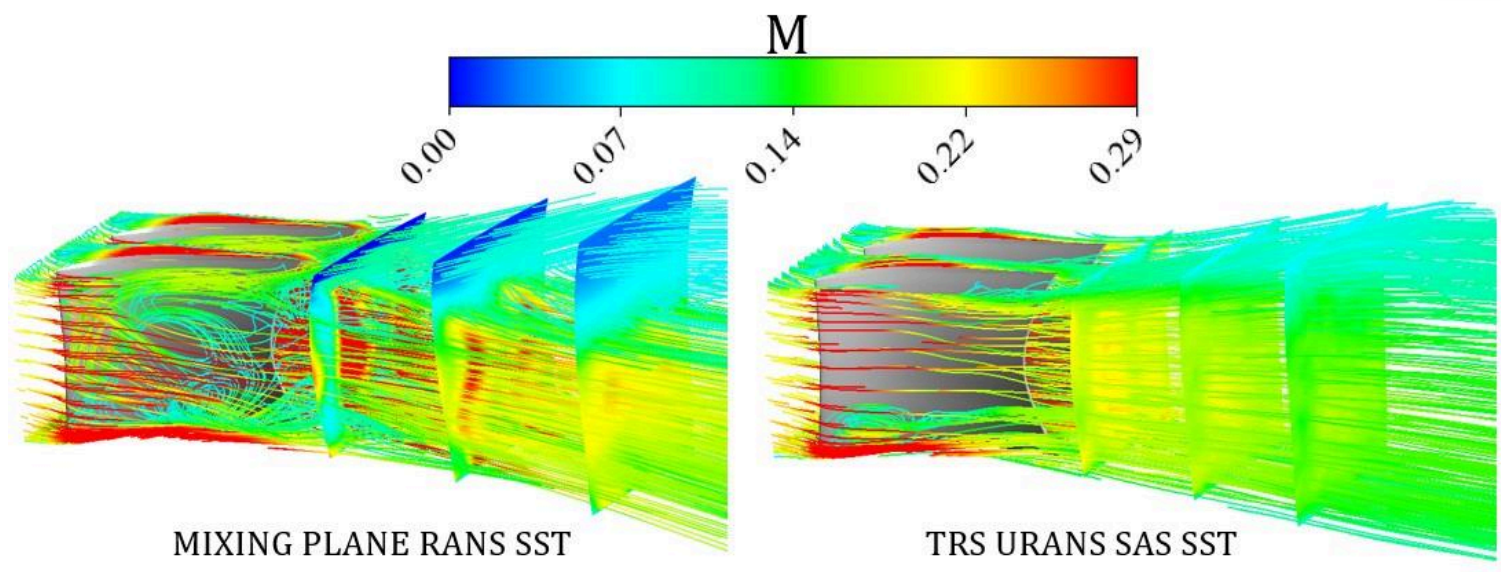

Figure 9 Difference in computed flow-fields from a steady RANS and a URANS SAS solution (time-averaged) for the compressor discharge. The RANS model predicts a strong re-circulation region near the top of the pre-diffuser, this is not present in time-averaged unsteady results. [Color online].

As evidenced in Figure 8, the bulk flow-fields predicted by both unsteady implementations (URANS SST and SAS SST), result in largely similar flow-fields. The SAS solution yields a more diffuse time-averaged result due to the presence of resolved turbulent structures. This yields a higher standard deviation of the velocity magnitude over the same period, as seen in Figure 10. This difference in statistics 
motivates the use of a scale resolving approach such as SAS to characterize any unsteady dynamics that may impact combustion performance.

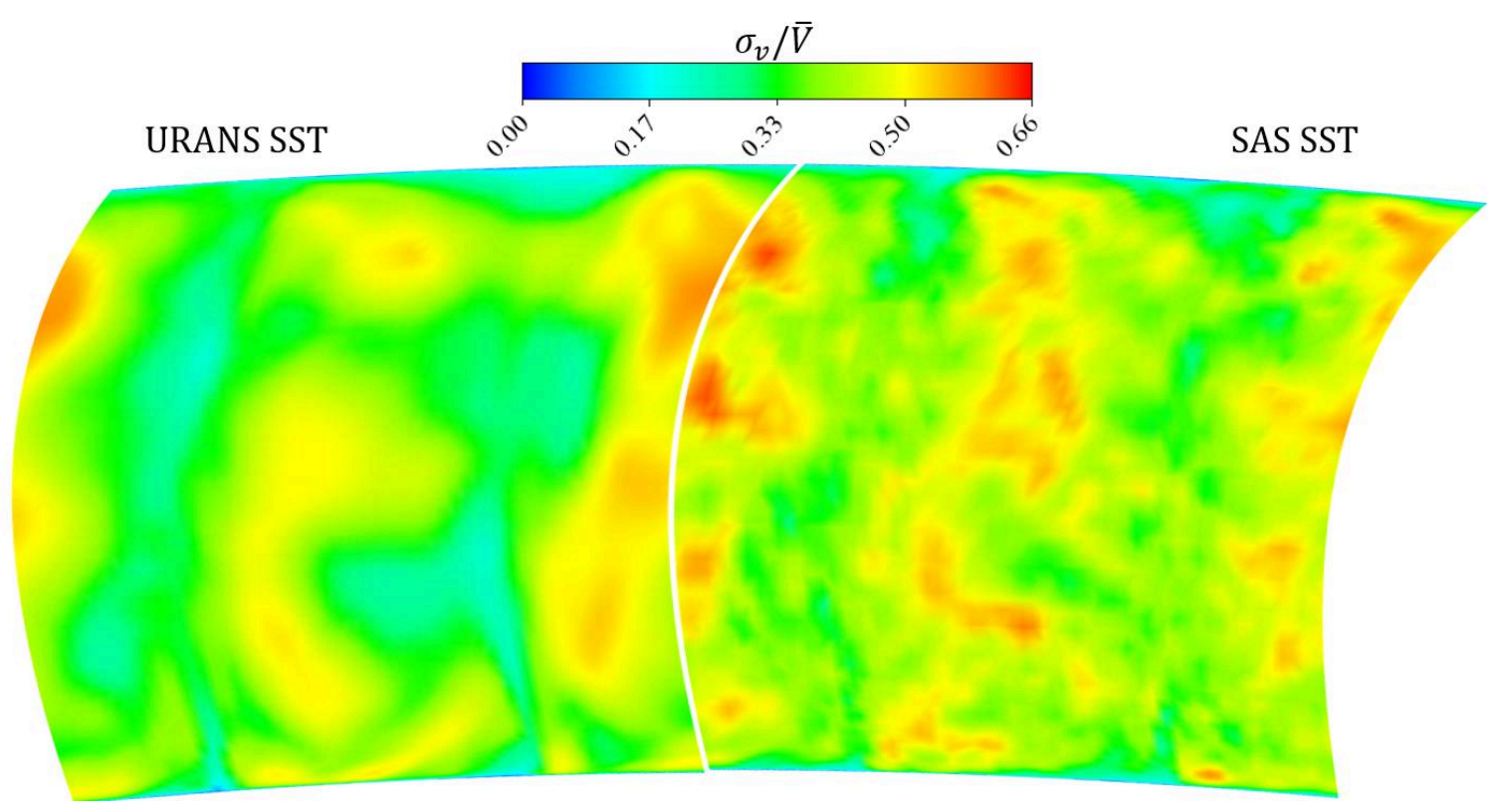

Figure 10 Velocity magnitude standard deviation at Discharge Plane with URANS SST and SAS SST, both using TRS interfaces for all stages. [Color online].

\section{Interface Modelling}

The modeling of stage interfaces also needs to be assessed, as downstream flowdynamics may depend on what occurs upstream. Transient Rotor-Stator (TRS) interfaces with a profile transformation may be used on all interfaces, with the profile transformation accounting for pitch differences between blade-rows. Alternatively, mixing planes may be used between all stages where the unsteady solution calculated at each blade row is then averaged circumferentially in between each blade row. A combination approach considers mixing planes only in the front stages and TRS in the last stage. The difference between the calculated flow fields can be seen in the time- 
averaged Mach and normalized static pressure at the discharge plane, as shown in

Figure 11. Figure 12 shows the velocity standard deviation at the same plane.

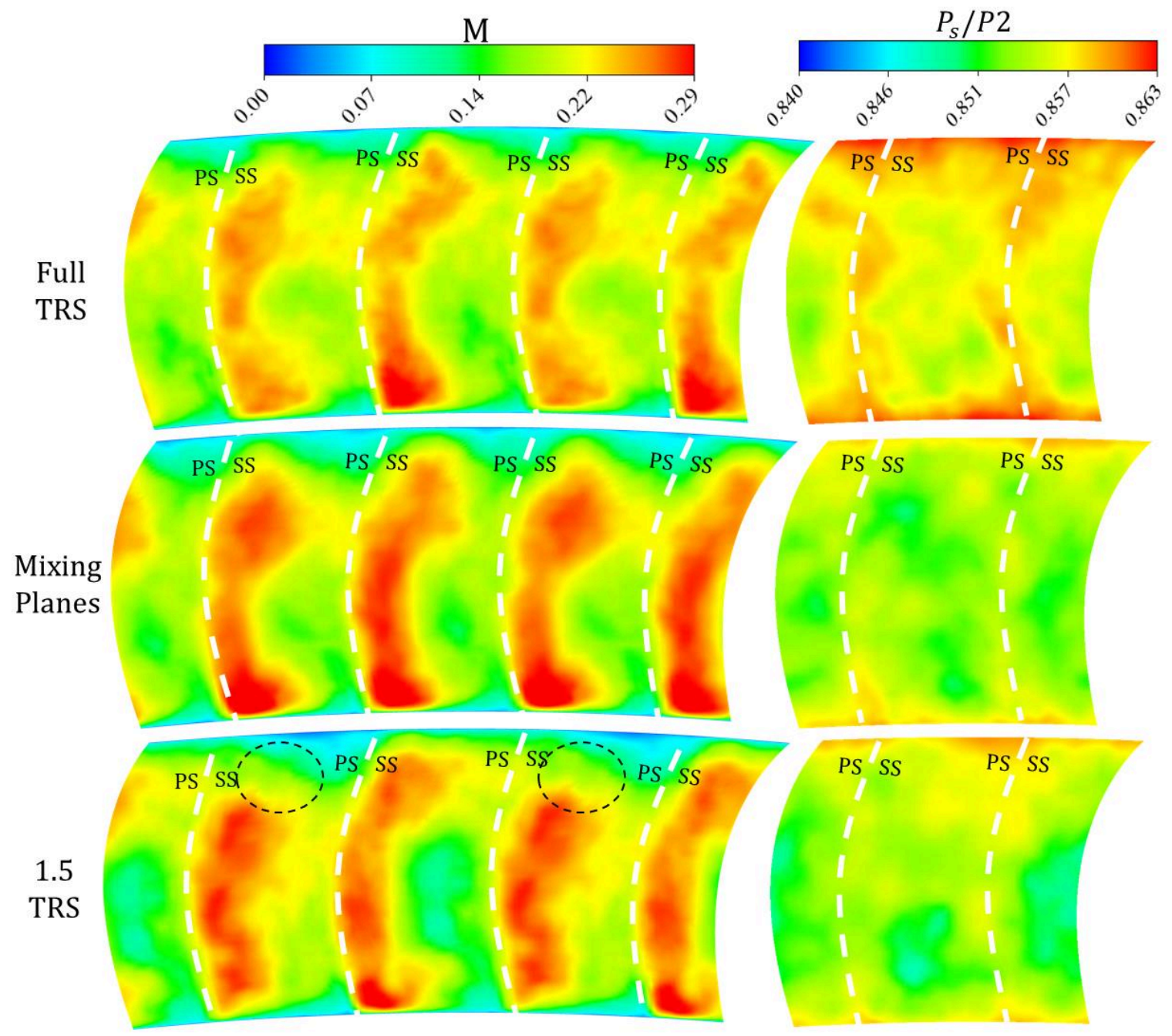

Figure 11 Time averaged Mach number (left) and normalized static pressure (right) at Discharge Plane with three different interface approaches. Top to bottom: TRS interfaces throughout, mixing planes throughout, TRS interfaces only in the last two interfaces. Dashed lines mark approximate trailing edge location. [Color online]. 


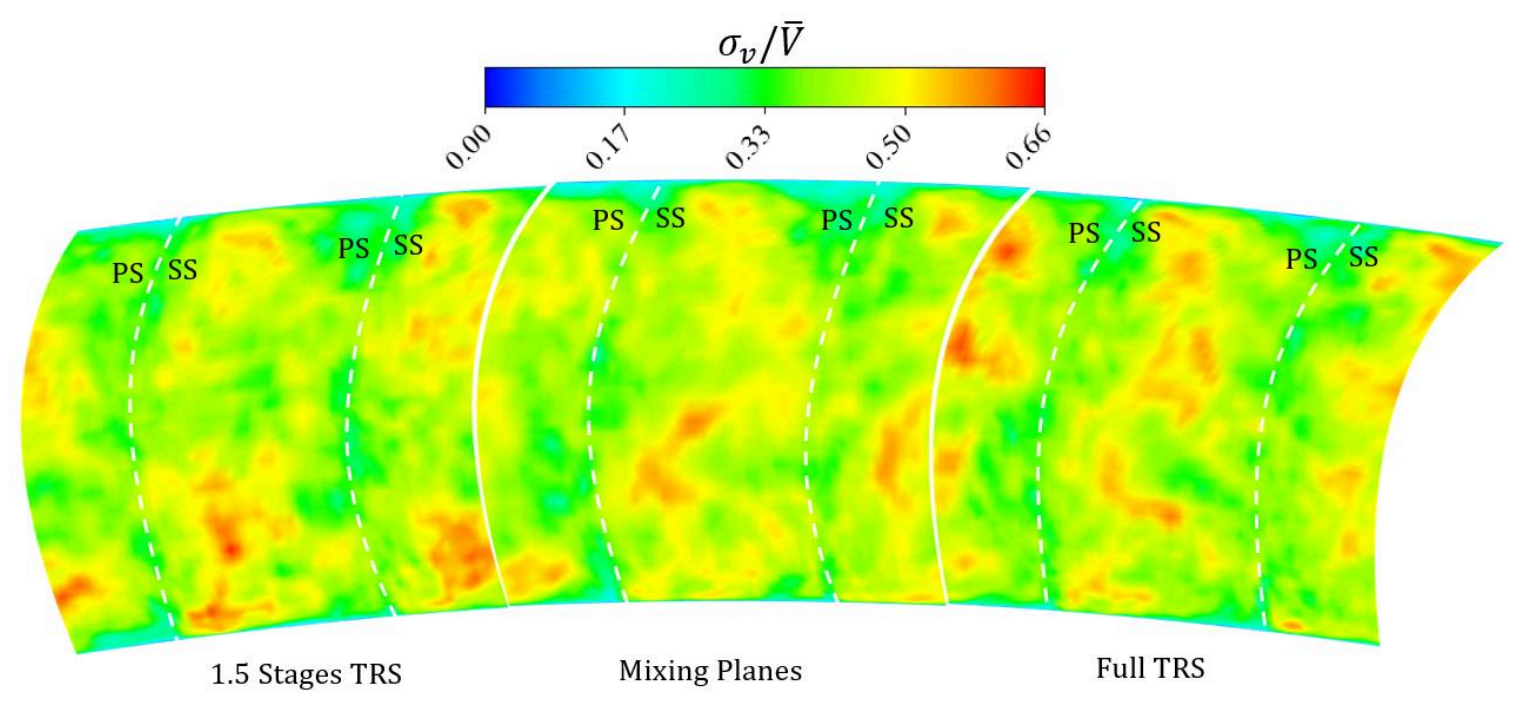

Figure 12 Velocity magnitude standard deviation at Discharge Plane with three different interface approaches. Left to right: TRS interfaces throughout, TRS interfaces only in the last two interfaces, and mixing planes throughout. Dashed lines mark approximate trailing edge location. [Color online].

The time averaged velocity magnitudes from the three approaches are seen to be broadly similar. As may be expected, slightly higher pressure loss is seen in the methods employing mixing planes versus the Full TRS implementation. This can be explained from the additional entropy generation incurred in a mixing process. As wakes are not instantaneously mixed out for the Full TRS case, less entropy is generated. An additional point to note is the highlighted region present in the 1.5 TRS case. As can be seen, the wakes from the two modelled stators are not identical. This can be explained from the relative circumferential location of the upstream stators, known as the clocking effect [9] , as shown in Figure 13. 


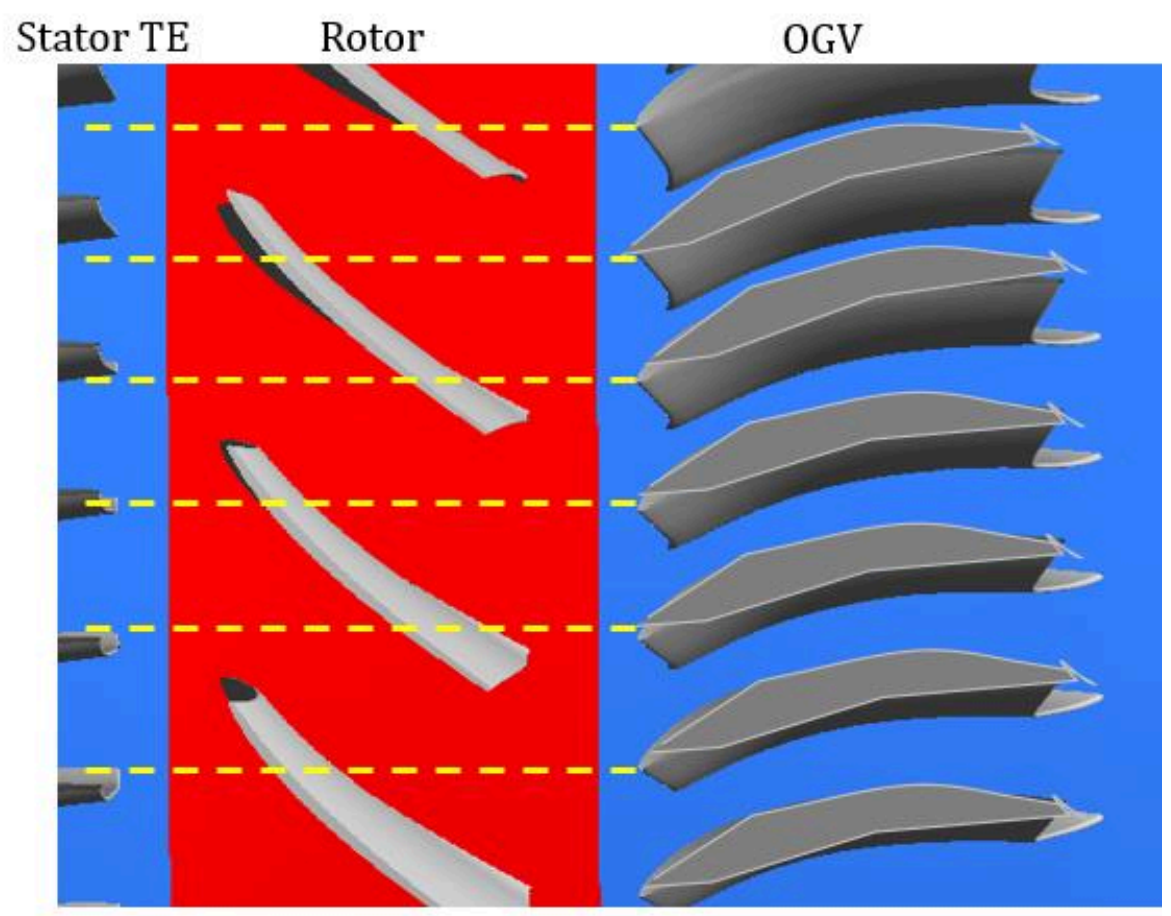

Figure 13 Relative azimuthal location of blading in last 3 blade rows. Geometry altered due to confidentiality. [Color online].

Since the OGVs do not line up with the upstream stators and a TRS interface is used, wakes from the upstream stator impact the time-averaged result at the discharge plane. Interestingly, this is only seen for the 1.5 TRS case and not the Full TRS approach. A possible explanation for this lies in the fact that, in the 1.5 TRS case, the upstream stator is presented with a circumferentially uniform flow-field from the mixing plane employed. In the Full TRS case however, the flow into this stator is circumferentially non-uniform and time-varying, promoting greater mixing in the stator passage.

Despite the differences, the time-averaged flow-fields are largely similar. This is an important result, as it entails that reduced order models may be employed to further investigate compressor discharge at windmill and similar operating conditions. The 
advantage of the mixing plane approach is that the difference in pitch between bladerow domains is automatically handled via the circumferential averaging. If TRS approaches are used, a profile transformation similar to what would be used in a frozen rotor model is used to scale fluxes between domains of unequal pitch. For cases where the pitch difference is large, multiple blades would need to be modelled to reduce the pitch ratio and reduce errors resulting from the scaling [17]. In the particular case modelled here, the last two blade rows are integral multiples of each other, so modelling two stator blades is enough to avoid any scaling to be performed in that stage. This may not be the general case. In the worst case scenario, a geometry may exist where the entire annulus may need to be modelled.

The sole use of mixing planes may be considered, even in an unsteady solution, if the unsteady dynamics within the blade-row are deemed to be dominant over the rotorstator interactions. In this case, the use of mixing planes throughout the entire compressor model has little effect on the flow statistics, but does have some effect on the turbulence parameters. Figure 14 shows the TKE spectrum measured downstream of the OGV. The depicted spectrums are the average of the spectrums obtained at five different radial locations as previously discussed. Three different spectrums are shown corresponding to the three different interface modelling approaches. All spectrums follow the power law in the inertial range as is theoretically expected from homogenous turbulence decay. No distinct peaks are seen, indicating broadband turbulence with no distinct structures. There is only some small difference in spectral power between the mixing plane formulation and the two TRS approaches towards higher wavenumbers. This would appear to indicate that 
some structures of smaller length scale may be present in the mixing plane approach when compared to the two TRS approaches. Figure 15 shows a comparison of the modelled TKE at the discharge plane for the three approaches. Higher levels of modelled turbulence are present in the mixing plane model. SAS resolves turbulence when it is large enough to be resolved by the underlying mesh, falling back on the URANS model otherwise. The fact that higher modelled turbulence appears in the mixing plane method aligns well with the slight difference in spectrums seen in Figure 14, indicating somewhat smaller turbulence length scales arising when the rotor stator interaction is not accounted for. The relevance of this difference in modelled turbulence depends on the application. For many studies requiring compressor discharge data, the mixing plane approach may be sufficient, while a single stage TRS implementation in the last stage may be considered more appropriate for cases when turbulence details are necessary. In any case, results shown here indicate that not much is gained from a whole compressor TRS implementation, pointing to the notion that the compressor discharge flow field under stabilized windmilling conditions is largely dictated by the flow dynamics of the last stage. 


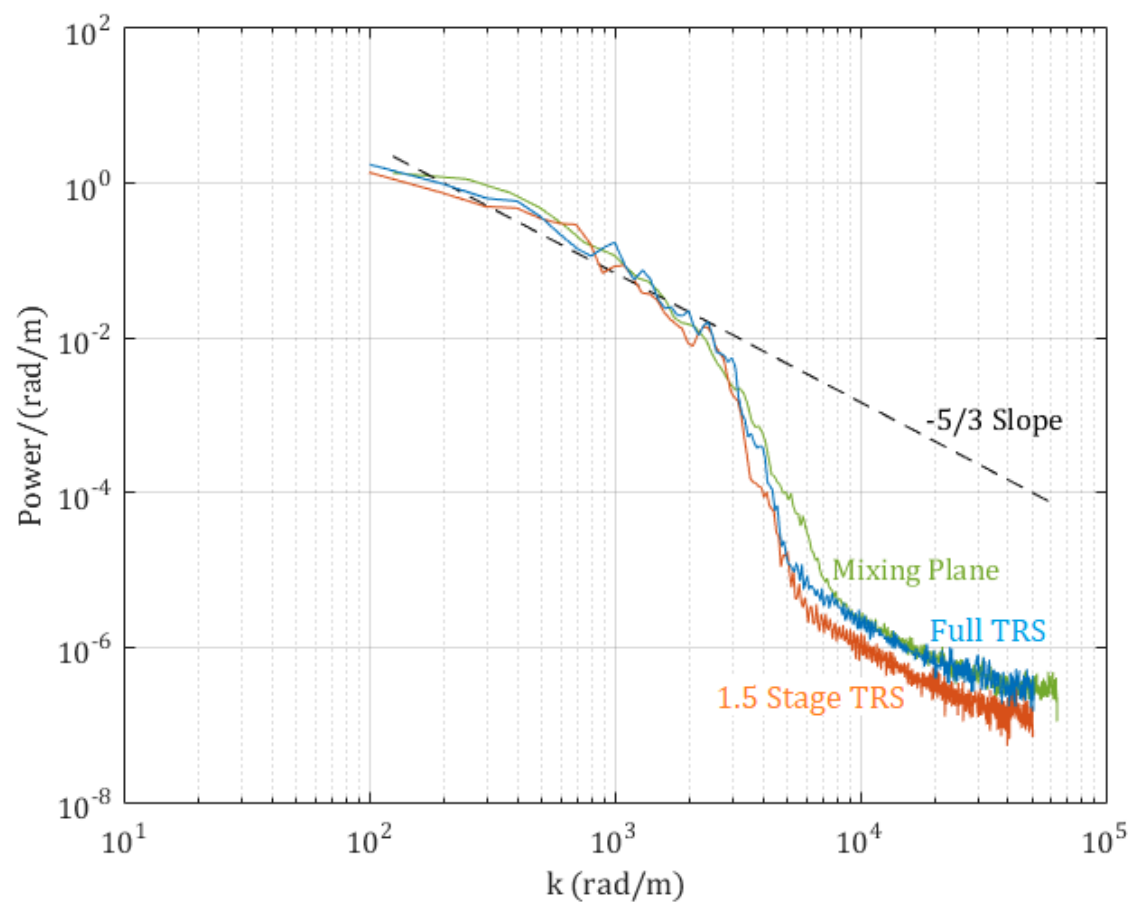

Figure 14 TKE spectrum for three different interface modelling approaches. Averaged spectrum from 5 evenly spaced radial locations downstream of the blade trailing edge, approximately at one third pitch. [Color online].
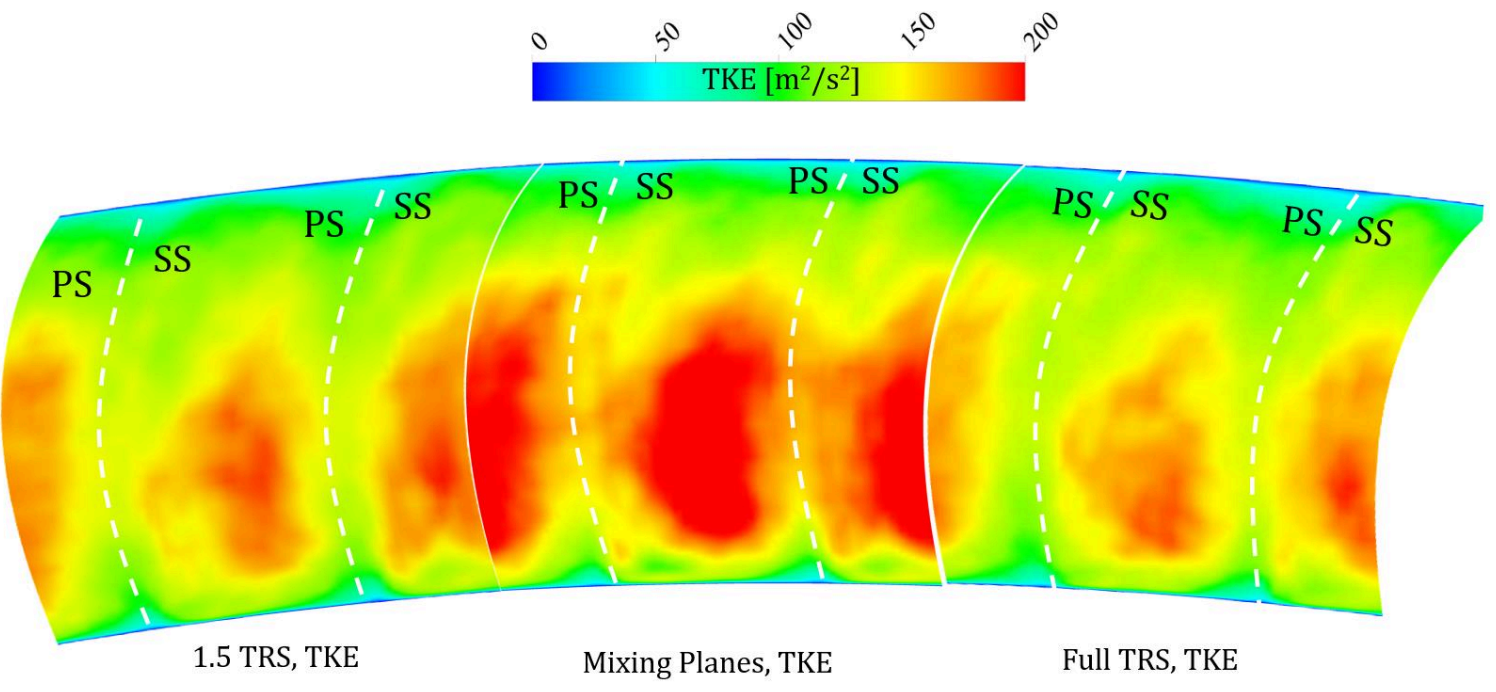

Figure 15 Time averaged modelled TKE at Discharge Plane for the 1.5 TRS, Mixing Plane, and Full TRS approaches. Dashed lines mark approximate trailing edge location. [Color online]. 


\section{Flow-field Description}

Normalized velocity components may be extracted from the Full TRS solution at the discharge plane, this is shown in Figure 16. As can be seen, the discharge flow during windmill is mostly axial, with little swirl.

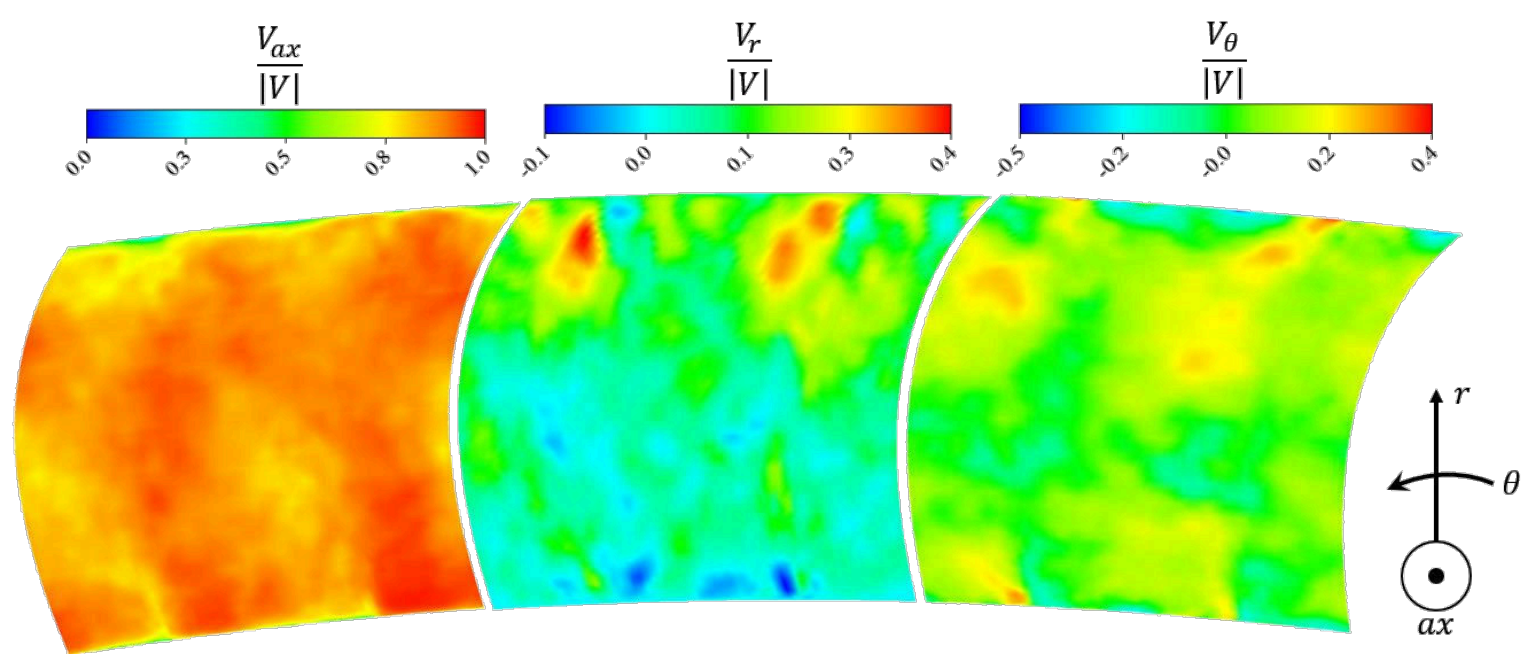

Figure 16 Normalized time-averaged velocity components at discharge plane. Obtained from full TRS solution. [Color online].

As previously mentioned, turbulent spectra downstream of the OGV appears to be broadband, with no distinct turbulent structures of note. This is more qualitatively investigated by looking at iso-surfaces of Q-criterion in the OGV domain, as shown in Figure 17. Turbulent structures with length scales approximating the leading edge diameter are shed from the leading edge, breaking up downstream before the discharge plane is reached. 


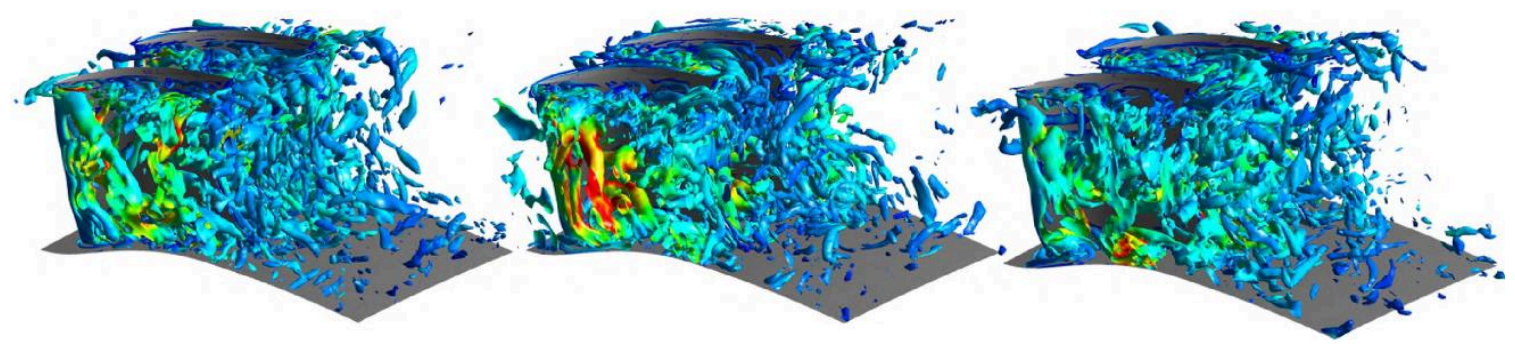

Figure 17 Contours of $Q$ criterion $\left(Q=5 \times 10^{\wedge} 8 \mathrm{~Hz}^{\wedge} 2\right)$ showing the formation of turbulent structures at the stator leading edge and their break-up downstream. Three different instances shown. [Color online].

As observed in Figure 17, turbulent structures created at the leading edge quickly break down into smaller scales throughout the passage, with no large structures remaining at the discharge plane. This results in a high degree of mixing and largely even time-averaged flow-field, with contours of turbulence kinetic energy (TKE) and turbulence eddy dissipation (TED) that reach a mixed-out condition half a bladechord downstream of the trailing edge. Likewise, losses mix-out in approximately the same distance, and this is shown in Figure 18 in terms of a normalized entropy generation coefficient ( $\widetilde{\Delta s}$ ), which is defined as the local specific entropy change from the domain inlet divided by the mass flow averaged change in entropy from inlet to outlet, as shown in Eq. 3. A value of 0 corresponds to a value of entropy corresponding to the mass-flow averaged inlet value, while a value of 1 corresponds to the same quantity at the outlet of the domain. Values at the outlet in Figure 18 are not exactly 1 due to it depicting a cascade view at $50 \%$ span, since higher entropy generation near end-walls is not shown in the figure.

$$
\widetilde{\Delta s}=\frac{\dot{m}_{\text {total }} \times s-\sum_{\text {in }}(\dot{m} \times s)}{\sum_{\text {out }}(\dot{m} \times s)-\sum_{\text {in }}(\dot{m} \times s)}
$$


The high degree of mixing yields a highly even flow-field in terms of turbulent quantities at the discharge plane, with only mildly higher values of TKE near the center of the passage, away from the trailing edges, due to the transported turbulence from the leading edge separation region. This is seen in Figure 18.
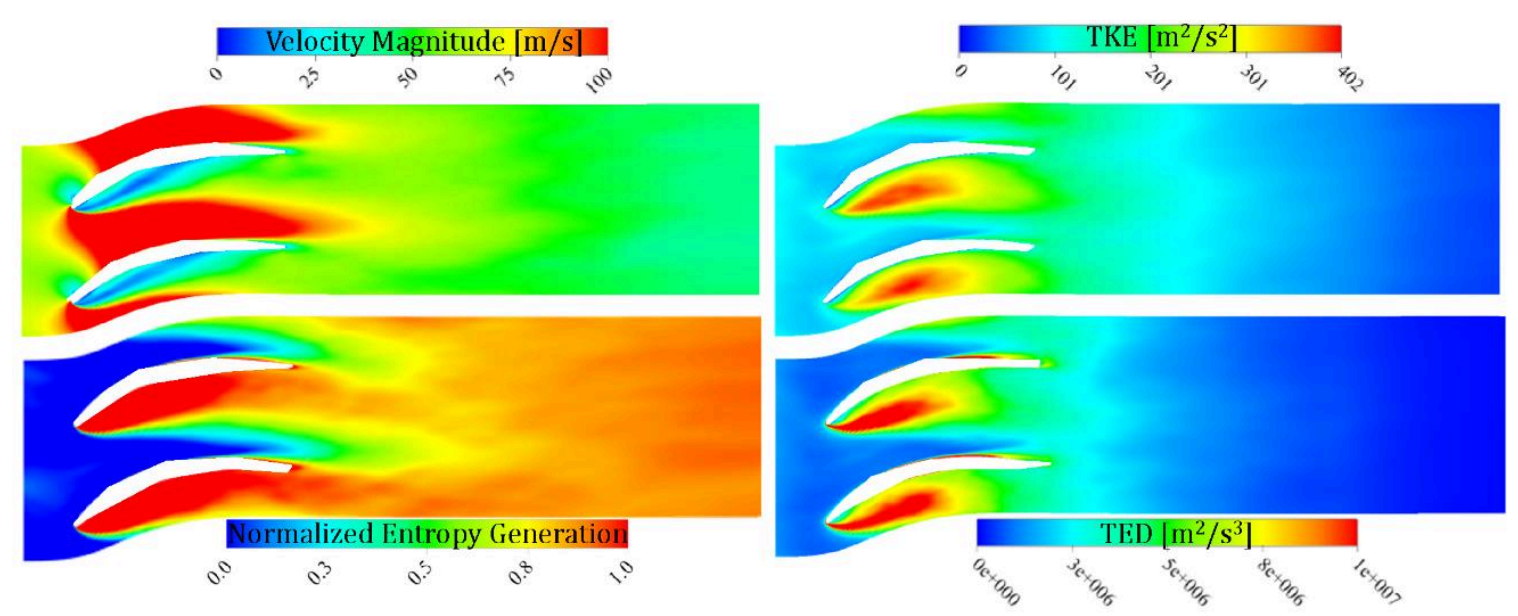

Figure 18 Time-averaged velocity magnitude, TKE, TED \& Normalized Entropy generation on cascade plane at $50 \%$ span. Geometry altered due to confidentiality. [Color online].

Bulk parameters at the discharge plane may be summarized in non-dimensional terms as shown in Table 3. Pressure and temperature are quoted as ratios of the same quantities at the inlet to the core compression system (turbofan engine core inlet).

Table 3 Compressor discharge representative parameters for altitude windmilling at top-left corner.

\begin{tabular}{|l|l|}
\hline Total Temperature [T3/T2] & 1.03 \\
\hline Total Pressure [P3/P2] & 0.90 \\
\hline Mass-flow Averaged Mach & 0.24 \\
\hline Turbulence Length Scale & 1 LE Diameter \\
\hline
\end{tabular}


An interesting dynamic to consider is the creation of corner vortices that get transported into the separation bubble on the blade pressure side, as these can contribute to enhance the mixing downstream of the stator blades via increased stream-wise vorticity. Similarly to the well-known dynamic of horseshoe vortex formation in turbine blades [28], the incoming boundary layer rolls-up into a saddle point near the stagnation point on the blade suction side. Two vortices get created which move in different directions. With the one closest to the neighbouring blade's pressure side feeding the separation region there. This is shown in Figure 19.
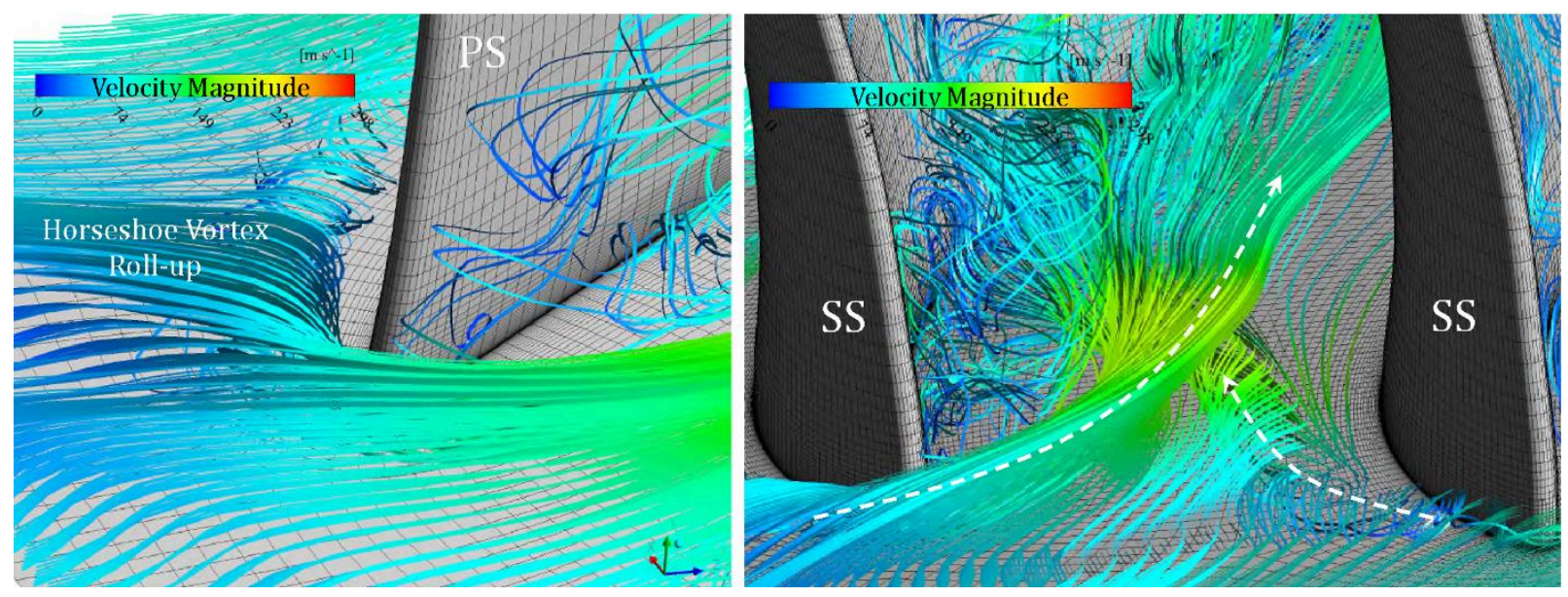

\section{Figure 19 Generation of corner vortex and transport of vortical structure into pressure side low pressure region. [Color online].}

Unlike in the horseshoe vortex dynamics of turbine blades, where a passage vortex is transported to the neighboring blade's suction side [28], the vortices created here both go to feed the pressure side separation region. This can be seen in Figure 20, where streamlines in the bottom $5 \%$ of span have been colored in different color bands to ease visualization. Figure 20 (left) shows a view of the OGV hub from below, looking radially out. In this view, different vortex features are identified with regards to how they are transported around the blading by the flow. Streamlines can be 
identified moving from the suction side stagnation point on one blade to the pressure side of the following blade. At the same time, many streamlines emanating from the same region can be seen to flow around the leading edge and into the separation region of their respective blade. Figure 20 (right) shows that the generated vortices are transported radially outwards within the separation region, eventually turning onto the stream-wise direction and contributing to stream-wise vorticity and the generation of secondary flows. Additionally, Figure 20 (right) shows the effect that incoming wakes from the up-stream rotor (identified by TKE greyscale contours) have on the pressure side separation region. The incoming levels of higher TKE reenergize the separated boundary layer, reducing the amount of separation.

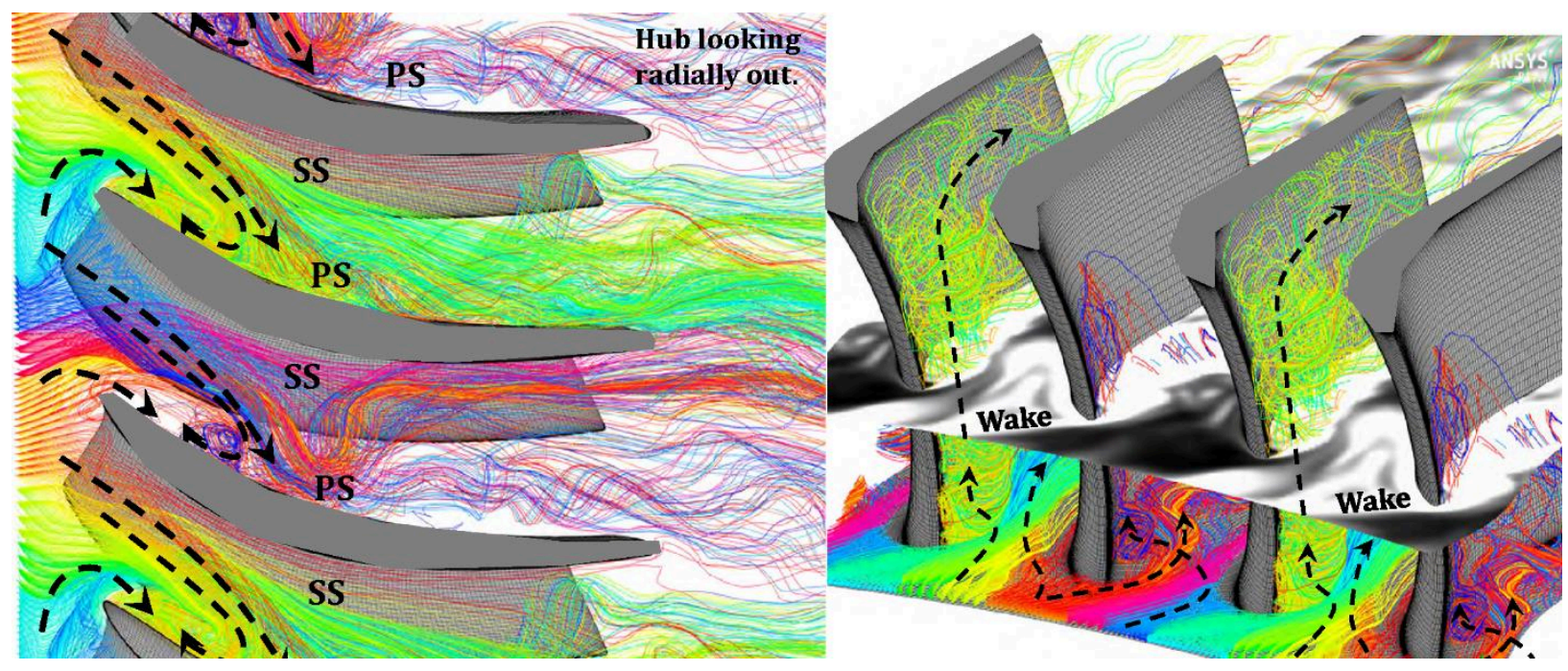

Figure 20 Transport of low momentum end wall flow into pressure side low pressure region and its radial development. White bands in right picture indicate higher TKE from upstream wake. Geometry altered due to confidentiality. [Color online].

Proper orthogonal decomposition applied to the discharge plane shows that the flow is dominated by the mean flow-field, which accounts for almost $50 \%$ of the resolved TKE. The next three most important modes account for much lower levels of TKE, 
indicating largely incoherent turbulence at the discharge plane. These modes are illustrated in Figure 21, while the distribution of $E_{\text {frac }}$ per POD mode for the first 20 modes is shown in Figure 22. The fact that the turbulence at this plane appears largely incoherent and dominated by the mean flow indicates that statistical flow descriptions, such as the time-averaged flow-field along with time-averaged turbulence quantities, could be used to appropriately prescribe the flow conditions into the combustor, without having to rely on a dynamical description. 


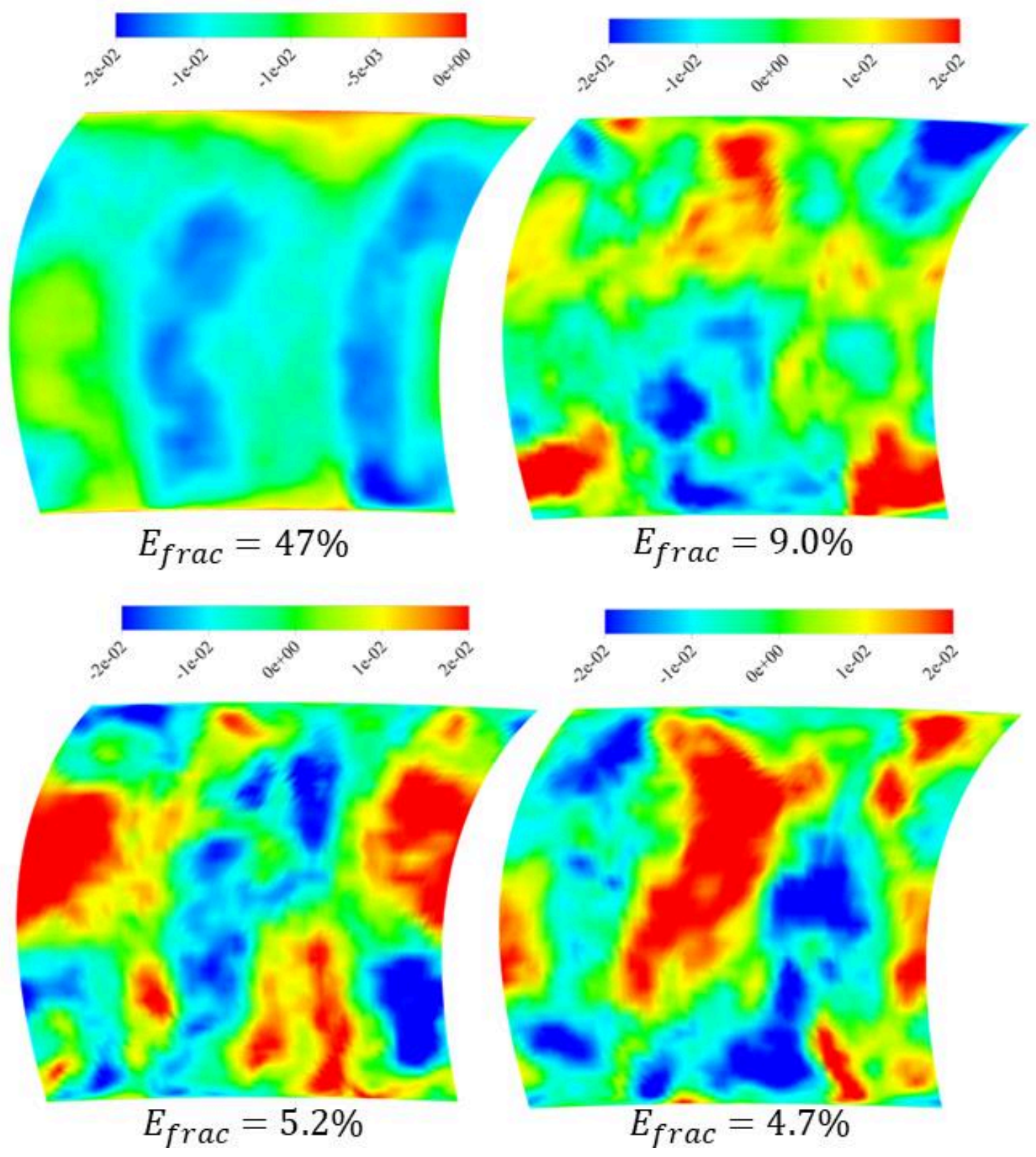

Figure 21 Four POD modes with highest TKE energy fraction (Efrac). [Color online]. 


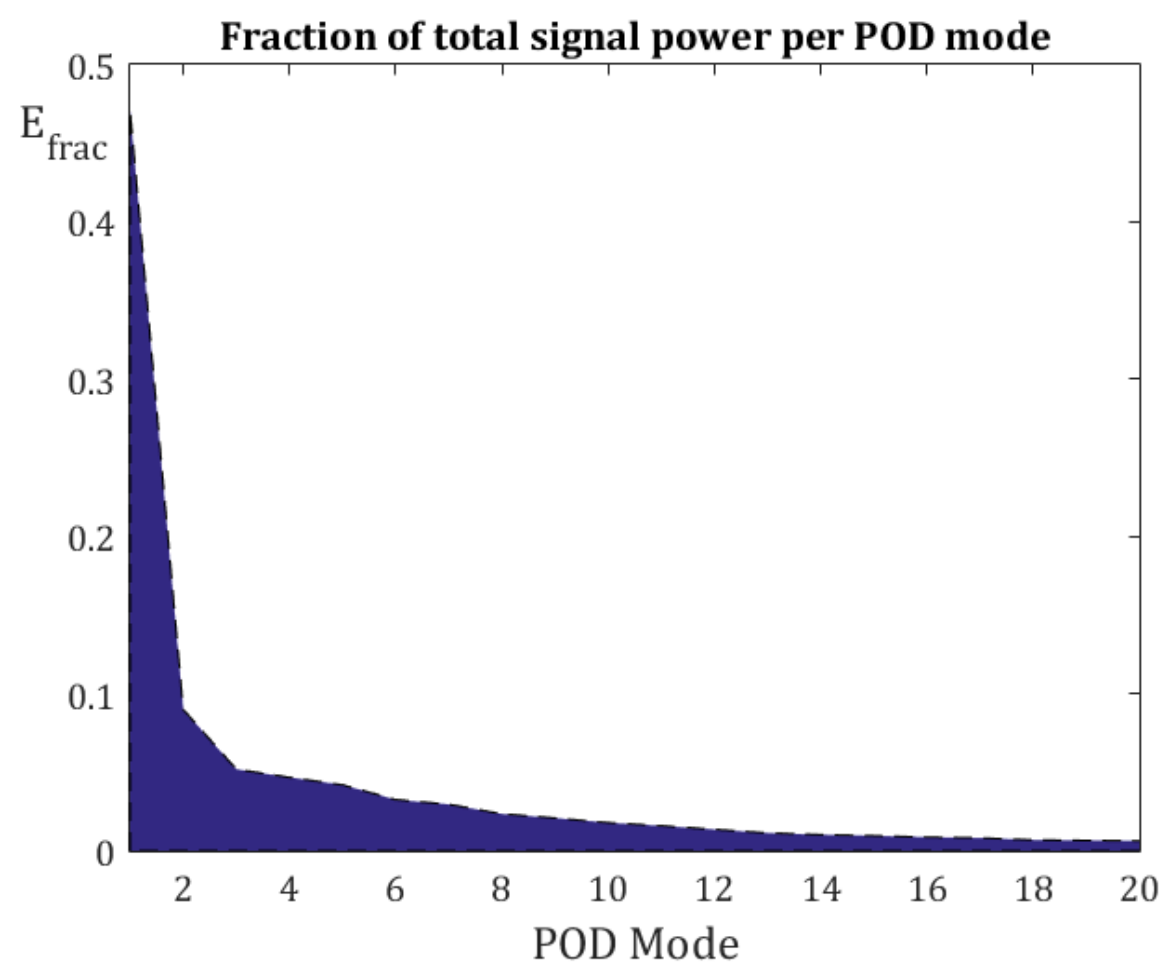

Figure 22 TKE fraction distribution of first 20 modes.

\section{Heat Soakage Effects}

Using the 1.5 TRS model, the same windmill case is run this time with a constant temperature applied on walls to represent the worst case heat transfer condition as previously explained. Figure 23 shows the difference in time averaged velocity magnitude due to the effect of heat soakage. As is seen, the flow field is qualitatively similar but with moderately higher velocity. The effect on the standard deviation is more pronounced, as shown in Figure 24. In terms of bulk parameters as reported in Table 3, T3/T2 has increased by $38 \%$, while no noticeable effect on $\mathrm{P} 3 / \mathrm{P} 2$ is 
observed. The mass flow decreases by $22 \%$ (a constant exit static pressure is prescribed).

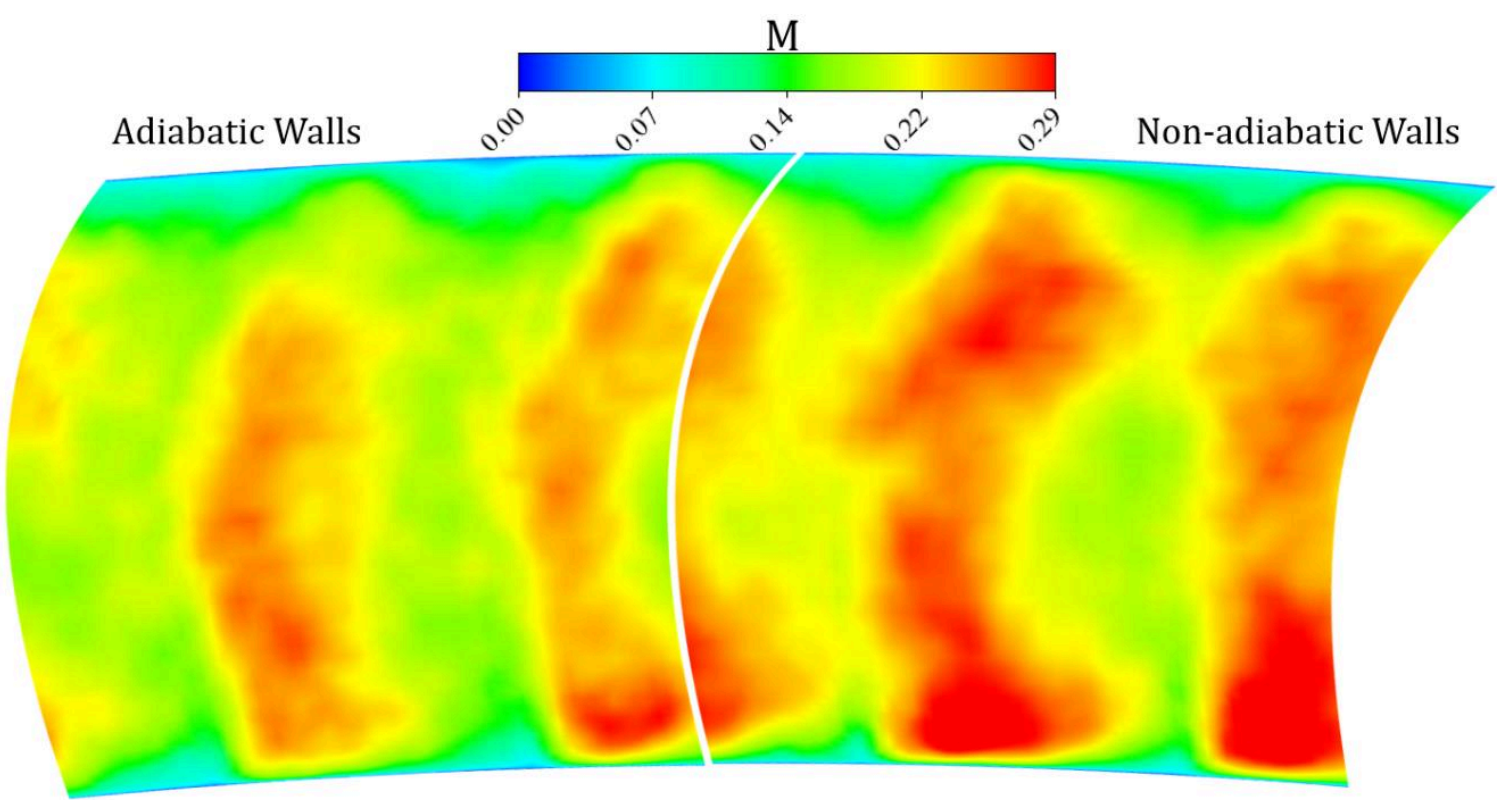

Figure 23 Calculated time-averaged Mach number at OGV outlet with and without adiabatic walls. [Color online].

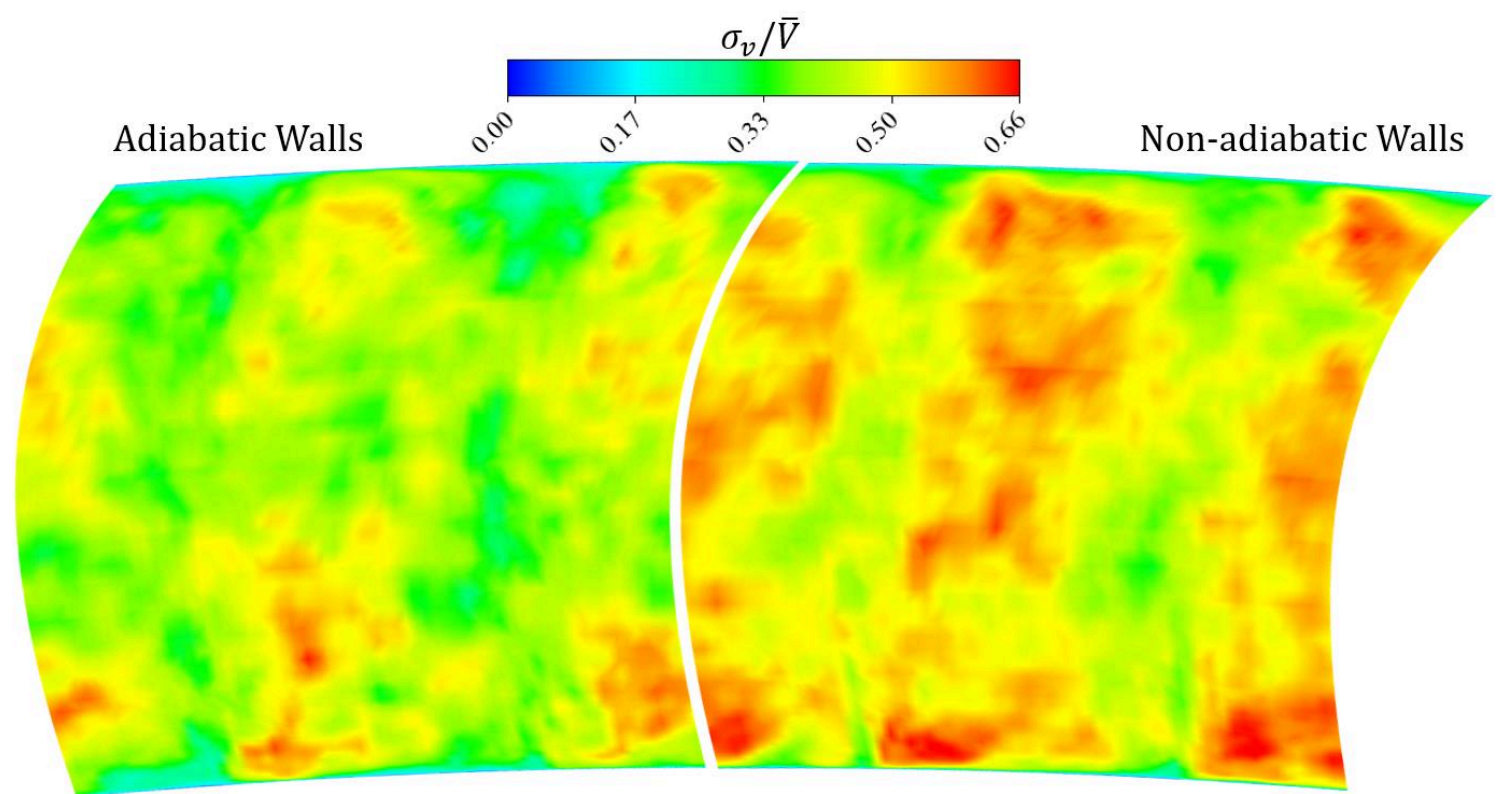

Figure 24 Calculated time-averaged velocity magnitude standard deviation at OGV outlet with and without adiabatic walls. [Color online]. 
The largest difference is observed in the turbulent quantities, as illustrated in Figure 25. The heat transfer causes larger fluctuations in velocity, causing larger values of turbulence kinetic energy. This can partly be explained from the increased importance of rotor-stator interaction, as the presence of rotor wakes upstream of the OGV is felt more strongly due to the temperature difference associated with the passage of hot blading.

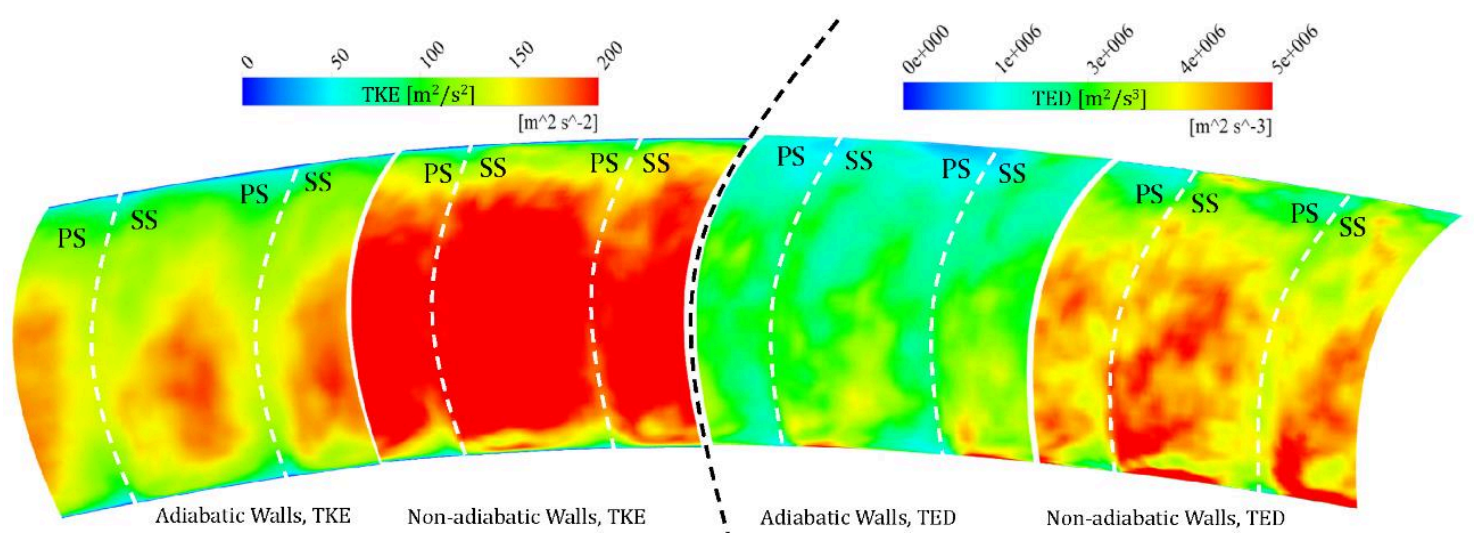

Figure 25 Calculated time-averaged turbulent quantities at OGV outlet with and without adiabatic walls. [Color online].

\section{Tip Clearance}

All models have included a tip clearance of $1 \%$ span. A 1.5 TRS model has also been run with $0 \%$ and $2 \%$ span tip clearances applied to the last stage rotor in order to assess its effect on the discharge flow-field. Figure 26 shows the difference in flowfields for the two extremes. Stream lines are shown colored by velocity magnitude. The difference between them is also shown. The increased vorticity due to the tip gap is clear, but the effects largely wash out when convected to the OGV, since the OGV pressure side separation is still the dominant feature there. This is seen in Figure 27, where the differences in TKE, TED and velocity standard deviation are seen to be 
negligible. Thus, while consideration should be given to the thermal state of the compressor when extracting the windmilling discharge flow-field, uncertainties in the values of tip clearance can be expected to be of negligible importance for windmill conditions.
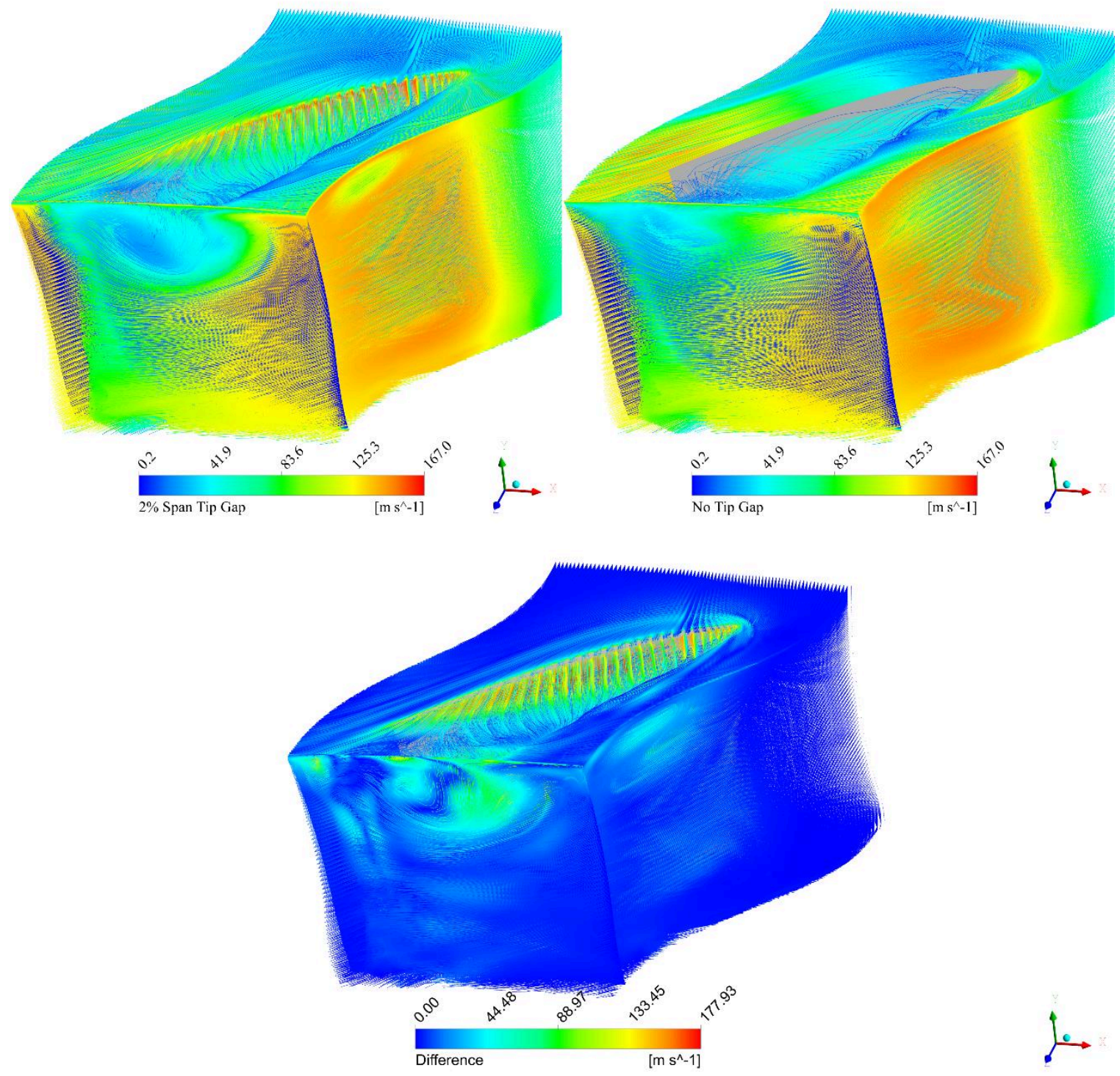

Figure 26 Difference in last stage rotor flow-field. Streamlines colored by velocity magnitude. [Color online]. 


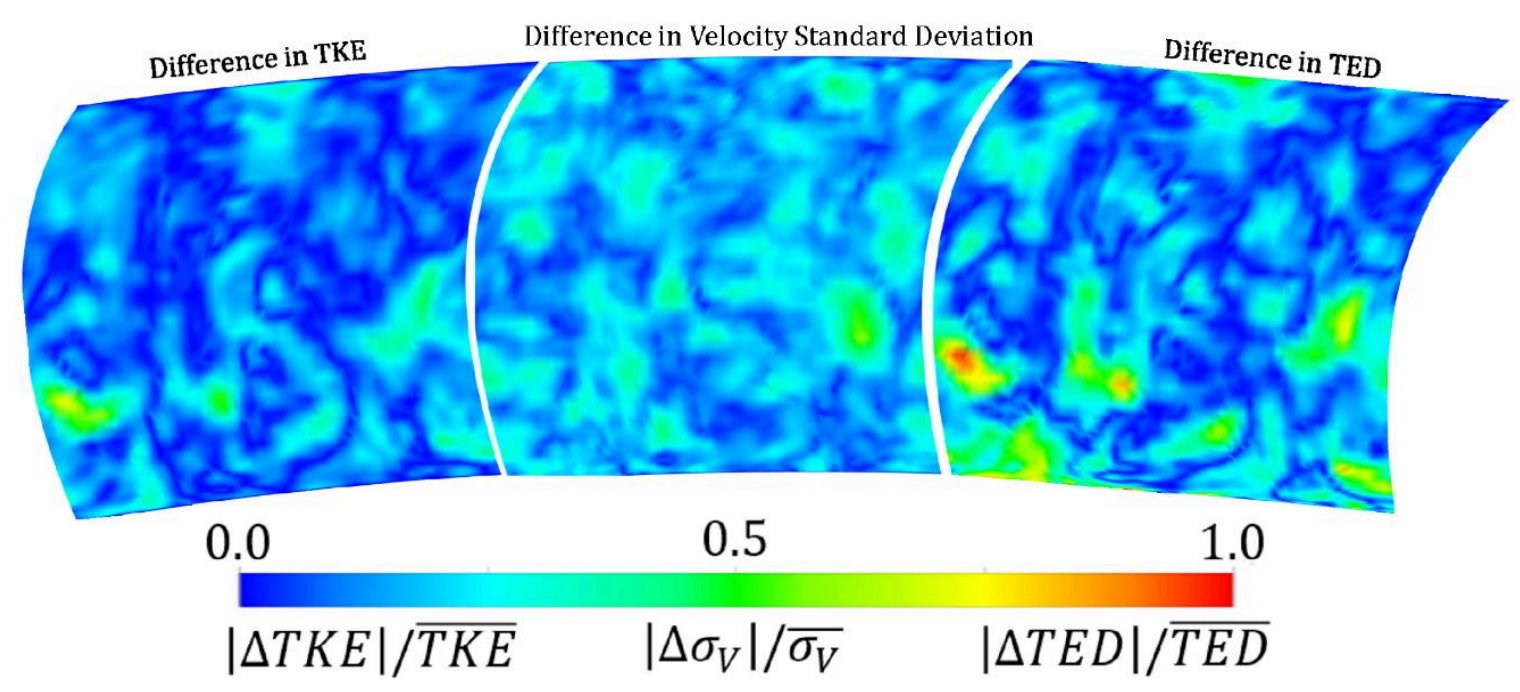

Figure 27 Difference in TKE, velocity standard deviation, and TED at discharge plane due to tip gaps in rotor upstream of OGV. Normalized by mass flow averaged value of the "No Tip Gap" case [Color online].

\section{CONCLUSIONS}

A modern core compressor has been modelled using scale adaptive simulation to extract the discharge flow-field that would be delivered to a combustor during a stabilized windmill at altitude. Different interface modelling approaches have been attempted to assess the effect these may have on results and a description of the flowfield has been provided. The following statements can be made:

- The flow-field at the compressor discharge is dominated by pressure side separation in the OGV.

- Transient rotor-stator interaction effects are thus of second importance.

- A mixing plane approach appears to appropriately characterize the timeaveraged flow-field at the discharge plane at the conditions studied. 
- A mixing plane solution will however result in slightly higher modelled TKE when using a SAS approach, indicating smaller turbulent length scales are obtained.

- The discharge flow-field is predominantly axial, with no coherent structures arising at the discharge plane and adequately described by the time-averaged statistics.

- A brief study has shown that the results may be sensitive to the use of diabatic vs. adiabatic boundary conditions on the compressor walls, while the effects of varying tip clearance are largely negligible.

These observations imply that future analyses looking at other windmilling or subidle operating points require an unsteady analysis, but may use a decoupled or reduced order approach to focus only on the flow-dynamics of the last stator without having to model blade-row domain interfaces throughout the machine. Experimental validation of these observations on interface modelling would be valuable.

The time-averaged statistics from URANS and SAS models, both employing SST, yield equivalent statistics. The well-developed turbulent flow-field present entails URANS approaches may be sufficient to capture representative time-averaged statistics. Flow-field details at the discharge plane have been provided to allow further analyses of combustion performance during altitude relight.

This study has focused on stabilized windmill relight, where the compressor may be considered to have reached thermal equilibrium and where the adiabatic assumption is appropriate. For quick-windmill relight studies however, where the compressor 
Author's Manuscript

may still be hot from operation near cruise, due consideration will need to be given to effect that heat transfer in the compressor may have on the combustor inlet conditions.

\section{ACKNOWLEDGMENT}

The authors thank Mark Stockwell and Richard Tunstall, of Rolls-Royce plc, for providing the data needed to carry out this work. The authors would like to thank Rolls-Royce plc for supporting this work and allowing its publication.

\section{FUNDING}

This work has received funding from the European Union (EU) Commission Horizon 2020 framework program under the CleanSky2 project PROTEUS, grant number 785349.

Due to confidentiality agreements with research collaborators, supporting data can only be made available to bona fide researchers subject to a non-disclosure agreement. 
Author's Manuscript

\section{NOMENCLATURE}

$E_{\text {frac }} \quad$ Fraction of turbulence kinetic energy captured by each POD mode.

KCAS Knots Calibrated Air Speed

$k_{u} \quad$ Unresolved (modelled) turbulence kinetic energy.

$k_{\text {total }} \quad$ Total turbulence kinetic energy.

LE $\quad$ Blade leading Edge.

$L_{v K} \quad$ Von Karman length scale.

M Mach number.

$\dot{m} \quad$ Mass flow.

$P \quad$ Total pressure.

POD Proper Orthogonal Decomposition.

PS $\quad$ Blade pressure side.

RANS Reynolds Averaged Navier Stokes.

$S \quad$ Mean strain rate.

SS Blade suction side. 
Author's Manuscript

SAS Scale Adaptive Simulation.

SST Shear Stress Transport.

St Strouhal number.

$S \quad$ Entropy

$T \quad$ Total temperature.

TED Turbulence Eddy Dissipation.

$\overline{T E D} \quad$ Mass-flow averaged Turbulence Eddy Dissipation at discharge plane.

TKE Turbulence Kinetic Energy.

$\overline{T K E} \quad$ Mass-flow averaged Turbulence Kinetic Energy at discharge plane.

TRS Transient Rotor Stator interface.

$U^{\prime \prime} \quad$ Second derivative of velocity flow-field.

URANS Unsteady Reynolds Averaged Navier Stokes.

V Velocity.

$\bar{V} \quad$ Area-averaged velocity magnitude at discharge plane.

$\widetilde{\Delta S} \quad$ Normalized entropy generation coefficient. 
Author's Manuscript

$\kappa$

$v$

$v_{t}$

$\sigma_{i}$

$\sigma_{v}$

$\overline{\sigma_{v}}$

$\Omega$

\section{Subscripts}

2

$a x$

in

$r$

out

$\theta$
Von Karman length scale constant.

Molecular viscosity.

Eddy viscosity.

Singular values from Singular Value Decomposition.

Velocity standard deviation.

Mass-flow averaged velocity standard deviation at discharge plane.

Compressor angular frequency in $[\mathrm{rad} / \mathrm{s}]$.
Core compression system inlet station (after fan).

Core compression system outlet station.

Axial direction

Domain inlet station.

Radial direction.

Domain outlet station.

Circumferential direction. 
Author's Manuscript 


\section{REFERENCES}

[1] NTSB. Crash of Pinnacle Airlines Flight 3701; Bombardier CL-600-2B19, N8396A; Jefferson City, Missouri October 14, 2004. Technical report, NTSB, (2007).

[2] NTSB. Loss of thrust in both engines after encountering a flock of birds and subsequent ditching on the Hudson river; US Airways Flight 1549; Airbus A320-214, N106US; Weehawken, New Jersey; January 15, 2009. Technical report, NTSB (2010).

[3] NTSB. Safety recommendation A-05-19 and -20. Technical report, NTSB, Aug. (2005)

[4] Pub. L No. CS-E910,1: Certification memorandum: Turbine engine relighting in flight. Technical report, European Aviation Safety Agency (2015)

[5] A. Neophytou, E. Mastorakos, E.S. Richardson, S. Stow, and M. Zedda, A practical model for the high-altitude relight of a gas turbine combustor. Seventh Mediterranean Combustion Symposium (MCS-7), Cagliari, Italy. 10 - 14 Sep (2011). 8 pp

[6] S.D. Stouffer, T. H. Hendershott, J. Colborn, J. R. Monfort, E. Corporan, P. Wrzesinski, and A. Caswell, Fuel Effects on Altitude Relight Performance of a Swirl Cup Combustor AIAA Scitech Forum (2020), https://doi.org/10.2514/6.2020-1882

[7] A. Andreini, T. Bacci, M. Insinna, L. Mazzei, S. Salvadori, Modelling strategies for the prediction of hot streak generation in lean burn aeroengine combustors, Aerospace Science and Technology, Volume 79 (2018), Pages 266-277, ISSN 1270-9638, https://doi.org/10.1016/i.ast.2018.05.030.

[8] N. Grech. Gas Turbine Sub-Idle Performance Modelling; Groundstart, Altitude Relight, and Windmilling. PhD thesis, Cranfield University (2013).

[9] F. Chen, Z. Gu, H. Lu, Z. Wang, Experimental study of stator clocking effects in an axial compressor, Chinese Journal of Aeronautics, Vol. 19, Iss. 4 (2006)

[10] A.D. Naidu, K. Vogel, and M. Fischer. A comparative study of transient blade row and blade count scaling approaches for numerical forced response analysis in a transonic turbine. In 12th European Conference on Turbomachinery Fluid Dynamics and Thermodynamics, ETC 2017 (2017). https://doi.org/10.29008/ETC2017-305

[11] ANSYS Inc. ANSYS CFX Modelling Guide. Technical report (2016). 
[12] G.A. Gerolymos, G.J. Michon, and J. Neubauer. Analysis and application of chorochronic periodicity in turbomachinery rotor/stator interaction computations. Journal of Propulsion and Power, 18(6):1139-1152 (2002). https://doi.org/10.2514/2.6065

[13] L. He. Fourier methods for turbomachinery applications. Progress in Aerospace Sciences, 46(8):329-341 (2010). 10.1016/j.paerosci.2010.04.001

[14] M.B. Giles. Calculation of unsteady wake/rotor interaction. Journal of Propulsion and Power, 4(4):356-362 (1988). https://doi.org/10.2514/3.23074

[15] L. E. Ferrer-Vidal, A. Iglesias-Pérez, V. Pachidis, Characterization of axial compressor performance at locked rotor and torque-free windmill conditions, Aerospace Science and Technology, Volume 101 (2020), 105846, ISSN 1270-9638, https://doi.org/10.1016/j.ast.2020.105846.

[16] J.D. Denton. Some limitations of turbomachinery CFD. In Proceedings of the ASME Turbo Expo, volume 7, pages 735-745 (2010).

[17] T. Biesinger, C. Cornelius, C. Rube, A. Braune, G. Schmid, R. Campregher, P.G. Godin, and L. Zori. Unsteady CFD methods in a commercial solver for turbomachinery applications. In Proceedings of the ASME Turbo Expo, volume 7, pages 2441-2452 (2010).

[18] P.G. Tucker. Computation of unsteady turbomachinery flows: Part 1 progress and challenges. Progress in Aerospace Sciences, 47(7):522-545 (2011) https://doi.org/10.1016/i.paerosci.2011.06.004

[19] P.G. Tucker. Computation of unsteady turbomachinery flows: Part 2-LES and hybrids. Progress in Aerospace Sciences, 47(7):546-569 (2011) https://doi.org/10.1016/i.paerosci.2011.07.002

[20] F.R. Menter, Best Practice: Scale-Resolving Simulations in ANSYS CFD, ANSYS Germany GmbH, 2012.

[21] F.R. Menter and Y. Egorov. A scale-adaptive simulation model using two equation models. pages 271-283 (2005), https://doi.org/10.2514/6.2005$\underline{1095}$

[22] F. R. Menter, Y. Egorov, The scale-adaptive simulation method for unsteady turbulent flow predictions, part 1: theory and model description, Flow Turbul. Combust. 85 (2010) 113-138. https://doi.org/10.1007/s10494-0109264-5 
[23] C. Y. Xu, T. Zhang, Y.Y. Yu, J.H. Sun, Effect of von Karman length scale in scale adaptive simulation approach on the prediction of supersonic turbulent flow, Aerospace Science and Technology, Volume 86 (2019), Pages 630-639, ISSN 1270-9638, https://doi.org/10.1016/j.ast.2019.01.030.

[24] M. Zhu, J. Teng, X. Qiang, J. Feng, J. Feng, Impact of hub gap leakage on stator endwall flow in an axial compressor stage with casing treatment, Aerospace Science and Technology, Volume 94 (2019), 105399, ISSN 1270-9638, https://doi.org/10.1016/j.ast.2019.105399.

[25] B. Šekutkovski, I. Kostić, A. Simonović, P. Cardiff, V. Jazarević, Threedimensional fluid-structure interaction simulation with a hybrid RANS-LES turbulence model for applications in transonic flow domain, Aerospace Science and Technology, Volume 49 (2016), Pages 1-16, ISSN 1270-9638, https://doi.org/10.1016/i.ast.2015.11.028.

[26] ANSYS CFX Theory Guide. Technical report, ANSYS Inc. (2016).

[27] S. Pope. Turbulent Flows. Cambridge University Press (2000).

[28] A. Iglesias Pérez. Scale-resolving simulations of compressor blading at negative incidences. Master's thesis, Cranfield University (2017)

[29] J. Lytle, G. Follen, C. Naiman, A. Evans, J. Veres, K. Owen, I. Lopez, Numerical Propulsion System Simulation (NPSS) 1999 Industry Review, NASA/TM2000-209795, NASA Glenn Research Center (2000)

[30] J.N. Kutz, S.L. Brunton, B.W. Brunton, J. L. Proctor, Dynamic Mode Decomposition: Data-Driven Modeling of Complex Systems, SIAM, (2016), ISBN: 9781611974492

[31] L.S. Langston. Secondary flows in axial turbines - a review. Annals of the New York Academy of Sciences, 934:11-26, (2001). https://doi.org/10.1111/j.1749-6632.2001.tb05839.x 
$2020-11-30$

Numerical modeling of core compressor discharge for a windmilling aero engine

Ferrer-Vidal, Luis E.

Elsevier

Ferrer-Vidal LE, Pachidis V. (2021) Numerical modeling of core compressor discharge for a windmilling aero engine. Aerospace Science and Technology, Volume 108, January 2021 , Article number 106365

https://doi.org/10.1016/j.ast.2020.106365

Downloaded from Cranfield Library Services E-Repository 